\title{
Universal volume bounds in Riemannian manifolds
}

\author{
Christopher B. Croke and Mikhail Katz
}

\begin{abstract}
In this survey article we will consider universal lower bounds on the volume of a Riemannian manifold, given in terms of the volume of lower dimensional objects (primarily the lengths of geodesics). By 'universal' we mean without curvature assumptions. The restriction to results with no (or only minimal) curvature assumptions, although somewhat arbitrary, allows the survey to be reasonably short. Although, even in this limited case the authors have left out many interesting results.
\end{abstract}

\section{Contents}

1. Introduction

2. Area and length of closed geodesics in 2 dimensions

3. Gromov's Filling Riemannian Manifolds

4. Systolic freedom for unstable systoles

5. Stable systolic and conformal inequalities

6. Isoembolic Inequalities

7. Acknowledgments

\section{Introduction}

In the present article, we will consider $n$-dimensional Riemannian manifolds $\left(X^{n}, \mathbf{g}\right)$, for the most part compact. We plan to survey the results and open questions concerning volume estimates that do not involve curvature (or involve it only very weakly). Our emphasis will be on open questions, and we intend the article to be accessible to graduate students who are interested in exploring these questions. In choosing what to include here, the authors have concentrated on results that have influenced their own work and on recent developments. In particular, we are only able to mention some of the highlights of M. Gromov's seminal paper [Gr83]. His survey devoted specifically to systoles appeared in [Gr96]. The interested reader is encouraged to explore those papers further, as well as his recent book [Gr99]. 
The estimates we are concerned with are lower bounds on the Riemannian volume (i.e. the $n$-dimensional volume), in terms of volume-minimizing lower dimensional objects. For example, one lower dimensional volume we will consider will be the infimum of volumes of representatives of a fixed homology or homotopy class. This line of research was, apparently, originally stimulated by a remark of René Thom, in a conversation with Marcel Berger in the library of Strasbourg University in the 1960's, not long after the publication of [Ac60, B161]. Having been told of the latter results (discussed in sections 3 and 1), Professor Thom reportedly exclaimed: "Mais c'est fondamental! [These results are of fundamental importance!]." Ultimately this lead to the so called isosystolic inequalities, $c f$. section 4. An intriguing historical discussion appears in section "Systolic reminiscences" [Gr99, pp. 271-272].

We will devote section 2 to the study of lower bounds on the area of surfaces (two dimensional manifolds) in terms of the length of closed geodesics. This is where the subject began, with the theorems of Loewner and $\mathrm{Pu}$, and where the most is known.

In section 3 we discuss some of the results and questions that come from [Gr83]. In particular, inequality (3.1), which provides a lower bound for the total volume in terms of an invariant called the Filling Radius, is one of the main tools used to obtain isosystolic inequalities in higher dimensions. We also discuss Gromov's notion of Filling Volume, as well as some of the open questions and recent results relating the volume of a compact Riemannian manifold with boundary, to the distances among its boundary points [B-C-G96, Cr01, C-D-S00, C-D, Iv02].

In section 4 , we see a collection of results that show that the inequalities envisioned in M. Berger's original question are often violated. Unless we are dealing with one-dimensional objects, such isosystolic inequalities are systematically violated by suitable families of metrics. This line of work was stimulated by M. Gromov's pioneering example (4.5), cf. [Gr96, Ka95, Pitte97, BabK98, BKS98, KS99, KS01, Bab02, Ka02].

Section 5 discusses how the systolic inequalities that we saw failed in Section 4, can in fact hold if we pass to stable versions. We survey the known results of this nature [Gr83, He86, BanK03, BanK2], and also examine the related conformal systolic invariants and their asymptotic behavior, studied in [BuSa94, Ka3].

In section 6 , we discuss bounds on the volume in terms of the injectivity radius of a compact Riemannian manifold. We discuss the (sharp) isoembolic inequality (6.3) of M. Berger, as well as the local version, i.e. volumes of balls, by Berger and the first author. We survey some of the extensions of these results, with an emphasis on the open questions and conjectures.

\section{Area and length of closed geodesics in 2 dimensions}

In this section, we will restrict ourselves to 2-dimensional Riemannian manifolds $\left(X^{2}, \mathbf{g}\right)$ and discuss lower bounds on the total area, area $(\mathbf{g})$, in term of the least length of a closed geodesic $L(\mathbf{g})$. In any dimension, the shortest loop in every nontrivial homotopy class is a closed geodesic. We will denote by $s y s \pi_{1}(\mathbf{g})$, the least length (often referred to as the "Systole" of $\mathbf{g}$ ) of a noncontractible loop $\gamma$ in a compact, non-simply-connected Riemannian manifold $(X, \mathbf{g})$ :

$$
\operatorname{sys}_{1}(\mathbf{g})=\min _{[\gamma] \neq 0 \in \pi_{1}(X)} \text { length }(\gamma) \text {. }
$$




\begin{tabular}{|l||l|l|l|}
\hline & $\sup _{\mathbf{g}} \frac{\text { sys } \pi_{1}(\mathbf{g})^{2}}{\operatorname{area}(\mathbf{g})}$ & numerical value & where to find it \\
\hline \hline$X=\mathbb{R} P^{2}$ & $=\frac{\pi}{2}[\mathbf{P u 5 2}, \mathbf{T a 9 2}]$ & $\approx 1.5707$ & $(2.5)$ \\
\hline infinite $\pi_{1}(X)$ & $<\frac{4}{3}[\mathbf{G r 8 3}]$ & $<1.3333$ & $(2.10)$ \\
\hline$X=\mathbb{T}^{2}$ & $=\frac{2}{\sqrt{3}}($ Loewner $)$ & $\approx 1.1547$ & $(2.3)$ \\
\hline$X=\mathbb{R} P^{2} \# \mathbb{R} P^{2}$ & $=\frac{\pi}{2^{3 / 2}}[$ Bav86, Sak88] & $\approx 1.1107$ & $(2.14)$ \\
\hline$X$ of genus 2 & $>(27-18 \sqrt{2})^{-\frac{1}{2}}$ & $>0.8047$ & $(2.8)$ \\
\hline$X$ of genus 3 & $\geq \frac{8}{7 \sqrt{3}}[\mathbf{C a 9 6}]$ & $>0.6598$ & section 5 \\
\hline$X$ of genus 4 & $>\frac{9 \sqrt{7}}{40}[\mathbf{C a 0 3}, \mathbf{C a 3}]$ & $>0.5953$ & section 5 \\
\hline$X$ of genus $s$ & $<\frac{64}{4 \sqrt{s}+27}[\mathbf{G r 8 3 , ~ K o 8 7}]$ & & $(2.9)$ \\
\hline
\end{tabular}

Figure 2.1. Values for optimal systolic ratio of surface $(X, \mathbf{g})$, in decreasing order

Hence $\operatorname{sys}_{1}(\mathbf{g}) \geq L(\mathbf{g})$. In this section (with the exception of section 4 where there is no homotopy) we will consider isosystolic inequalities of the following form: sys $\pi_{1}(\mathbf{g})^{2} \leq$ const area $(\mathbf{g})$. This leads naturally to the notion of the systolic ratio of an $n$-dimensional Riemannian manifold $(X, \mathbf{g})$, which is defined to be the scaleinvariant quantity $\frac{s y s \pi_{1}(\mathbf{g})^{n}}{\operatorname{vol}_{n}(\mathbf{g})}$. We also define the optimal systolic ratio of a manifold $X$ to be the quantity

$$
\sup _{\mathbf{g}} \frac{s y s \pi_{1}(\mathbf{g})^{n}}{\operatorname{vol}_{n}(\mathbf{g})}
$$

where the supremum is taken over all Riemannian metrics $\mathbf{g}$ on the given manifold $X$. Note that in the literature, the reciprocal of this quantity is sometimes used instead. In this section, we will discuss the optimal systolic ratio for $\mathbb{T}^{2}, \mathbb{R} P^{2}$ (section 1), and the Klein bottle $\mathbb{R} P^{2} \# \mathbb{R} P^{2}$ (section 5 ). These are the only surfaces for which the optimal systolic ratio is known. However, upper bounds for the systolic ratio, namely isosystolic inequalities, are available for all compact surfaces (section 3 ) except of course for $S^{2}$ (but see section 4). Figure 2.1 contains a chart showing the known upper and lower bounds on the optimal systolic ratios of surfaces.

1. Inequalities of Loewner and $\mathbf{P u}$. The first results of this type were due to C. Loewner and P. Pu. Around 1949, Carl Loewner proved the first systolic inequality, $c f$. [Pu52]. He showed that for every Riemannian metric $\mathbf{g}$ on the torus $\mathbb{T}^{2}$, we have

$$
\operatorname{sys} \pi_{1}(\mathbf{g})^{2} \leq \frac{2}{\sqrt{3}} \operatorname{area}(\mathbf{g})
$$


while a metric satisfying the boundary case of equality in (2.3) is necessarily flat, and is homothetic to the quotient of $\mathbb{C}$ by the lattice spanned by the cube roots of unity.

Two distinct optimal generalisations of (2.3) are available, $c f .(5.17)$ and (5.14). It follows from Gromov's estimate (2.9) that aspherical surfaces satisfy Loewner's inequality (2.3) if the genus is bigger than 50. It is an open question whether the genus assumption can be removed, but for genus up to 30, some information can be deduced from the Buser-Sarnak inequality (5.8).

We give a slightly modified version of M. Gromov's proof [Gr96], using conformal representation and Cauchy-Schwartz, of Loewner's theorem for the 2-torus. We present the following slight generalisation: there exists a pair of closed geodesics on $\left(\mathbb{T}^{2}, \mathbf{g}\right)$, of respective lengths $\lambda_{1}$ and $\lambda_{2}$, such that

$$
\lambda_{1} \lambda_{2} \leq \frac{2}{\sqrt{3}} \operatorname{area}(\mathbf{g})
$$

and whose homotopy classes form a generating set for $\pi_{1}\left(\mathbb{T}^{2}\right)=\mathbb{Z} \times \mathbb{Z}$.

Proof. The proof relies on the conformal representation $\phi: \mathbb{T}_{0} \rightarrow\left(\mathbb{T}^{2}, \mathbf{g}\right)$, where $\mathbb{T}_{0}$ is flat. Here $\phi$ may be chosen in such a way that $\left(\mathbb{T}^{2}, \mathbf{g}\right)$ and $\mathbb{T}_{0}$ have the same area. Let $f$ be the conformal factor of $\phi$.

Let $\ell_{0}$ be any closed geodesic in $\mathbb{T}_{0}$. Let $\left\{\ell_{s}\right\}$ be the family of geodesics parallel to $\ell_{0}$. Parametrize the family $\left\{\ell_{s}\right\}$ by a circle $S^{1}$ of length $\sigma$, so that $\sigma \ell_{0}=$ area $\left(\mathbb{T}_{0}\right)$. Thus $\mathbb{T}_{0} \rightarrow S^{1}$ is a Riemannian submersion. Then area $\left(\mathbb{T}^{2}\right)=\int_{\mathbb{T}_{0}} J a c_{\phi}=$ $\int_{\mathbb{T}_{0}} f^{2}$. By Fubini's theorem, area $\left(\mathbb{T}^{2}\right)=\int_{S^{1}} d s \int_{\ell_{s}} f^{2} d t$. By the Cauchy-Schwartz inequality,

$$
\operatorname{area}\left(\mathbb{T}^{2}\right) \geq \int_{S^{1}} d s \frac{\left(\int_{\ell_{s}} f d t\right)^{2}}{\ell_{0}}=\frac{1}{\ell_{0}} \int_{S^{1}} d s\left(\text { length } \phi\left(\ell_{s}\right)\right)^{2} .
$$

Hence there is an $s_{0}$ such that area $\left(\mathbb{T}^{2}\right) \geq \frac{\sigma}{\ell_{0}}$ length $\phi\left(\ell_{s_{0}}\right)^{2}$, so that length $\phi\left(\ell_{s_{0}}\right) \leq$ $\ell_{0}$. This reduces the proof to the flat case. Given a lattice in $\mathbb{C}$, we choose a shortest lattice vector $\lambda_{1}$, as well as a shortest one $\lambda_{2}$ not proportional to $\lambda_{1}$. The inequality is now obvious from the geometry of the standard fundamental domain for the action of $P S L(2, \mathbb{Z})$ in the upper half plane of $\mathbb{C}$.

We record here a slight generalization of Pu's theorem from [Pu52]. The generalization follows from Gromov's inequality (2.10). Namely, every surface $(X, \mathbf{g})$ which is not a 2-sphere satisfies

$$
\operatorname{sys}_{1}(\mathbf{g})^{2} \leq \frac{\pi}{2} \operatorname{area}(\mathbf{g}),
$$

where the boundary case of equality in (2.5) is attained precisely when, on the one hand, the surface $X$ is a real projective plane, and on the other, the metric $\mathbf{g}$ is of constant Gaussian curvature.

In both Loewner's and Pu's proofs, the area of two pointwise conformal metrics were compared to the minimum lengths of paths in a fixed family of curves (the family of all non contractible closed curves). This is sometimes called the conformal-length method and analyzed in [Gr83, section 5.5]. C. Bavard [Bav92a] was able to show that in a given conformal class, on any $n$-dimensional manifold, there is at most one metric with maximum systolic ratio, and was able to give a characterization of such metrics. 
We conjecture a generalisation, 2.7, of Pu's inequality (2.5). Let $S$ be a nonorientable surface and let $\phi: \pi_{1}(S) \rightarrow \mathbb{Z}_{2}$ be a homomorphism from its fundamental group to $\mathbb{Z}_{2}$, corresponding to a map $\hat{\phi}: S \rightarrow \mathbb{R} P^{2}$ of absolute degree one. We define the "1-systole relative to $\phi$ ", denoted $\phi s y s_{1}(\mathbf{g})$, of a metric $\mathbf{g}$ on $S$, by minimizing length over loops $\gamma$ which are not in the kernel of $\phi$, i.e. loops whose image under $\phi$ is not contractible in the projective plane:

$$
\operatorname{sis}_{1}(\mathbf{g})=\min _{\phi([\gamma]) \neq 0 \in \mathbb{Z}_{2}} \text { length }(\gamma) .
$$

The question is whether this systole of $(S, \mathbf{g})$ satisfies the following sharp inequality, related to Gromov's inequality $(*)_{\text {inter }}$ from $[\mathbf{G r 9 6}, 3$. C.1], see also $[$ Gr99, Theorem 4.41].

Conjecture 2.7. For any nonorientable surface $S$ and map $\hat{\phi}: S \rightarrow \mathbb{R} P^{2}$ of absolute degree one, we have $\phi$ sys $_{1}(\mathbf{g})^{2} \leq \frac{\pi}{2}$ area $(\mathbf{g})$.

In the above, the ordinary 1-systole of Pu's inequality (2.5) is replaced by the one relative to $\phi$. The example of the connected sum of a standard $\mathbb{R} P^{2}$ with a little 2 -torus shows that such an inequality would be optimal in every topological type $S$. Conjecture 2.7 is closely related to the the filling area of the circle (Conjecture 3.8). Given a filling $X^{2}$, one identifies antipodal points on the boundary circle to get a nonorientable surface $S$. One can define a degree one map $f$ from $S$ to $\mathbb{R} P^{2}$ by taking all points outside of a tubular neighborhood of the original boundary circle to a point. This gives a natural choice of $\phi$ above which relates the two conjectures, $c f$. Remarks $(e),\left(e^{\prime}\right)$ following [Gr83, Theorem 5.5.B'].

2. A surface of genus 2 with octahedral Weierstrass set. The optimal systolic ratio in genus 2 is unknown. Here we discuss a lower bound for the optimal systolic ratio in genus 2. The example of M. Berger (see [Gr83, Example 5.6.B'], or [Be83]) in genus 2 is a singular flat metric with conical singularities. Its systolic ratio is 0.6666 , which is not as good as the two examples we will now discuss.

C. Bavard [Bav92b] and P. Schmutz [Sch93, Theorem 5.2] identified the hyperbolic genus 2 surface with the optimal systolic ratio among all hyperbolic genus 2 surfaces. The surface in question is a triangle surface $(2,3,8)$. It admits a regular hyperbolic octagon as a fundamental domain, and has 12 systolic loops of length $2 x$, where $x=\cosh ^{-1}(1+\sqrt{2})$. It has sys $\pi_{1}=2 \log (1+\sqrt{2}+\sqrt{2+2 \sqrt{2}})$, area $4 \pi$, and systolic ratio 0.7437 .

This ratio can be improved by a singular flat metric $\mathbf{g}_{\mathcal{O}}$, described below. Note that it is an Hadamard space in a generalized sense, i.e. a CAT(0) space.

Start with a triangulation of the real projective plane with 3 vertices and 4 faces, corresponding to the octahedral triangulation of the 2 -sphere. The fact that the metric is pulled back from $\mathbb{R} P^{2}$ is significant, to the extent that if we can show that an extremal metric satisfies this, we would go a long way toward identifying it explicitly.

Every conformal structure on the surface of genus 2 is hyperelliptic [FK92, Proposition III.7.2], i.e. it admits a ramified double cover over the 2-sphere with 6 ramification points, called Weierstrass points. We take the 6 vertices of the regular octahedral triangulation, corresponding to the Riemann surface which has an equation of the form

$$
y^{2}=x^{5}-x
$$


This produces a triangulation of the genus 2 surface $\Sigma_{2}$ consisting of 16 triangles. If each of them is flat equilateral with side $x$, then the total area is $16\left(\frac{1}{2} x^{2} \sin \frac{\pi}{3}\right)=$ $4 \sqrt{3} x^{2}$. Meanwhile, sys $\pi_{1}=2 x$, corresponding to the inverse image of an edge under the double cover $\Sigma_{2} \rightarrow S^{2}$. The systolic ratio is $\frac{(2 x)^{2}}{4 \sqrt{3} x^{2}}=\frac{1}{\sqrt{3}}=0.5773$, which is not as good as the Bavard-Schmutz example.

Notice that the systolic loop remains locally minimizing if the angle of the triangle with side $x$ is decreased from $\frac{\pi}{3}$ to $\frac{\pi}{4}$ (and the space remains $\operatorname{CAT}(0)$ ). Therefore we replace the flat equilateral triangles by singular flat equilateral "triangles", whose singularity at the center has a total angle of $\frac{9 \pi}{4}=2 \pi\left(1+\frac{1}{8}\right)$, while the angle at each of the vertices is $\frac{\pi}{4}$. Its barycentric subdivision consists of 6 copies of a flat right angle triangle, denoted $\angle\left(\frac{x}{2}, \frac{\pi}{8}\right)$, with side $\frac{x}{2}$ and adjacent angle $\frac{\pi}{8}$. Notice that this results in a smooth ramification point (with a total angle of $2 \pi$ ) in the ramified cover. We thus obtain a decomposition of $\Sigma_{2}$ into 96 copies of the triangle $\measuredangle\left(\frac{x}{2}, \frac{\pi}{8}\right)$.

The resulting singular flat metric $\mathbf{g}_{\mathcal{O}}$ on $\Sigma_{2}$ (here $\mathcal{O}$ stands for "octahedron") has 16 singular points, with a total angle $\frac{9 \pi}{4}$ around each of them, 4 of them over each center of a face of the $\mathbb{R} P^{2}$ decomposition. This calculation is consistent with M. Troyanov's [Tro86] Gauss-Bonnet formula $\sum_{\sigma} \alpha(\sigma)=2 s-2$, where $s$ is the genus, where the cone angle at singularity $\sigma$ is $2 \pi(1+\alpha(\sigma))$.

Dually, the metric $\mathbf{g}_{\mathcal{O}}$ can be viewed as glued from six flat regular octagons, centered on the Weierstrass points. The hyperelliptic involution is the 180 degree rotation on each of them. The 1-skeleton projects to that of the inscribed cube in the 2-sphere. The systolic ratio of the resulting metric $\mathbf{g}_{\mathcal{O}}$ is

$$
\begin{aligned}
\frac{\left({\text { sys } \left.\pi_{1}\left(\mathbf{g}_{\mathcal{O}}\right)\right)^{2}}_{\operatorname{area~}\left(\mathbf{g}_{\mathcal{O}}\right)}\right.}{} & =\frac{(2 x)^{2}}{96 \text { area }\left(\triangle\left(\frac{x}{2}, \frac{\pi}{8}\right)\right)} \\
& =\frac{x^{2}}{24\left(\frac{1}{2}\left(\frac{x}{2}\right)^{2} \tan \frac{\pi}{8}\right)} \\
& =(3 \sqrt{3-2 \sqrt{2}})^{-1} \\
& =0.8047 .
\end{aligned}
$$

The genus 2 case is further investigated in [Ca3].

3. Gromov's area estimates for surfaces of higher genus. The earliest work on isosystolic inequalities for surfaces of genus $s$ is by R. Accola [Ac60] and C. Blatter [B161]. Their bounds on the optimal systolic ratio went to infinity with the genus, cf. (5.8). J. Hebda [He82] and independently Yu. Burago and V. Zalgaller [B-Z80, B-Z88] showed that for $s>1$ the optimal systolic ratio is bounded by 2 (which is not as good as (2.5), which came later). M. Gromov [Gr83, p. 50] (cf. [Ko87, Theorem 4, part (1)]) proved a general estimate which implies that if $\Sigma_{s}$ is a closed orientable surface of genus $s$ with a Riemannian metric, then

$$
\frac{\text { sys } \pi_{1}\left(\Sigma_{s}\right)^{2}}{\operatorname{area}\left(\Sigma_{s}\right)}<\frac{64}{4 \sqrt{s}+27} .
$$

Thus, as expected, the optimal systolic ratio goes to 0 as the genus goes to infinity (see (5.9) for the correct asymptotic behavior). 
Another helpful estimate is found in [Gr83, Corollary 5.2.B]. Namely, every aspherical closed surface $(\Sigma, \mathbf{g})$ admits a metric ball $B=B_{p}\left(\frac{1}{2} s y s \pi_{1}(\mathbf{g})\right) \subset \Sigma$ of radius $\frac{1}{2} s y s \pi_{1}(\mathrm{~g})$ which satisfies

$$
\operatorname{sys}_{1}(\mathbf{g})^{2} \leq \frac{4}{3} \operatorname{area}(B) .
$$

Furthermore, whenever a point $x \in \Sigma$ lies on a two-sided loop which is minimizing in its free homotopy class, the metric ball $B_{x}(r) \subset \Sigma$ of radius $r \leq \frac{1}{2}$ sys $\pi_{1}(\mathrm{~g})$ satisfies the estimate $2 r^{2}<\operatorname{area}\left(B_{x}(r)\right)$.

4. Simply connected and noncompact surfaces. One of the motivating questions for this section is from [Gr83, p. 135], see also problem 87 in [Ya82] (or [S-Y94]).

Question 2.11. For an $n$-dimensional compact manifold $X$, is there a constant $C(X)$ such that for every Riemannian metric $\mathbf{g}$ on $X$, we have

$$
\operatorname{vol}(\mathbf{g}) \geq C(X) L(\mathbf{g})^{n},
$$

where $L(\mathbf{g})$ is the length of the shortest nontrivial closed geodesic.

This is still open for many manifolds $X, e . g$. for $X=S^{n}, n \geq 3$. One could also ask the (stronger) question whether $C(X)$ depends only on $n$. Since $s y s \pi_{1}(\mathbf{g}) \geq L(\mathbf{g})$, upper bounds on the optimal systolic ratio give upper bounds on the constant $C(X)$ in (2.12). Thus we have a positive answer for all closed surfaces except for the two-sphere, $S^{2}$, which of course can have no nontrivial systolic inequalities. However, Question 2.11 does have an affirmative answer in this case. It was shown in $\left[\mathbf{C r} 88 \mathrm{~B}\right.$ ] that every metric $\mathbf{g}$ on $S^{2}$ satisfies the bound

$$
\frac{1}{31^{2}} L(\mathbf{g})^{2} \leq \operatorname{area}(\mathbf{g}) \text {. }
$$

Another estimate in that paper was $L(\mathbf{g}) \leq 9 D(\mathbf{g})$, where $D(\mathbf{g})$ represents the diameter. Neither of these constants are best possible, and both have been improved recently [Mae94, N-R02, Sab1]. The best known bounds are $L(\mathbf{g}) \leq 4 D(\mathbf{g})$ and $\frac{1}{64} L(\mathbf{g})^{2} \leq$ area $(\mathbf{g})$. The conjectured best constant $C\left(S^{2}\right)$ in $(2.12)$ for the 2 -sphere (suggested to the first author by E. Calabi) is $\frac{1}{2 \sqrt{3}}$, attained by the singular metric obtained by gluing two equilateral triangles along their edges.

A natural way to find closed geodesics on a non simply connected closed Riemannian manifold is to look for the shortest curve in a nontrivial homotopy class, as we have done in our consideration of $s y s \pi_{1}(\mathbf{g})$. However, when $\pi_{1}(X)$ is trivial, the standard technique is to used minimax arguments on nontrivial families of curves. For example, G. Birkhoff [Bi27] considered 1-parameter families of closed curves starting and ending in point curves, which pass over $S^{2}$ in the sense that the induced map from $S^{2}$ to $S^{2}$ has nonzero degree. He found a closed geodesic on $S^{2}$ by taking minimum over all these families of the maximum length curve in the family. These minimax geodesics are stationary in the sense that they are critical points (but not necessarily local minima) for the length functional on the space of curves. Thus one can find a short closed geodesic by finding such a family where every curve in the family is short. This idea played an important part in the proof of (2.13) above.

Instead of homotopy classes we could consider homology classes (leading to the notion of $\operatorname{sys}_{1}(X) c f$. (4.1)). That is, we could look at 1-cycles of minimal mass in 
nontrivial homology classes. When $H_{1}(X)$ is trivial one can instead use a minimax method on 1-cycles, similar to the one described in the previous paragraph, to get stationary 1-cycles (also see section 1). In some important cases these also turn out to be closed geodesics but in any event they are natural objects. The basis for this is the work Almgren [Al62] and Pitts [Pitts81, Theorem 4.10], who get stationary varifolds (in all dimensions) via minimax techniques. The case of 1-cycles (where things are easier) was exploited by Calabi and Cao [CaCa92] in their proof that the shortest closed geodesic on a convex surface is simple. They use the fact that on $S^{2}$ this minimax technique produces a closed geodesic. The estimates of A. Nabutovsky, R. Rotman, and S. Sabourau [N-R02, Sab1] improving (2.13) mentioned above exploited these techniques, $c f$. (3.6).

Although, as mentioned above, Question 2.11 is still open for general metrics on $S^{n}$, if one considers only convex hypersurfaces of $\mathbb{R}^{n+1}$ then such a result was shown independently in [Tre85] and [Cr88B]. The sharp constants are still not known in this case.

Finally one can ask Question 2.11 for noncompact surfaces and complete metrics (of finite area). In fact, the question has a positive answer for all surfaces. Most of the cases were dealt with as a special case of [Gr83, Theorem $4.4 \mathrm{~A}]$, while the other cases (the plane and the cylinder) were dealt with in $[\mathbf{C r} 88 \mathbf{B}]$.

5. Optimal surfaces, existence of optimal metrics, fried eggs. In the case of $\mathbb{T}^{2}$ or $\mathbb{R} P^{2}$, we saw in (2.3) and (2.5) that there is a particular smooth metric which achieves the optimal systolic ratio. In general this will not be the case unless one admits metrics with singularities. In fact, due to Gromov's compactness theorem $\left[\mathbf{G r 8 3}\right.$, theorem 5.6. $\left.\mathbf{C}^{\prime}\right]$, there will always be a singular metric achieving the maximal systolic ratio. Such metrics (as well as those that have locally maximal systolic ratio) are called "extremal isosystolic metrics". See [Gr83] or [Ca96] for a description of these singular metrics.

For the Klein bottle, C. Bavard [Bav86] (also see [Sak88]) found the maximal systolic ratio:

$$
\operatorname{sys}_{1}(\mathbf{g})^{2} \leq \frac{\pi}{2 \sqrt{2}} \operatorname{area}(\mathbf{g}) .
$$

He also identified the extremal isosystolic metric achieving it. This metric is singular, and is built out of two möbius strips of constant curvature +1 (where the central curve has length $\pi$, and width is $\frac{\pi}{2}$ ). They are then glued together along their boundaries. The metric is singular since the geodesic curvatures of both boundaries point outward.

An extensive study of such extremal metrics was undertaken by E. Calabi [Ca96]. He derived a number of properties of such extremal metrics using variation methods. For points in such a space at least two systoles must pass through each point. (We slightly abuse terminology by referring to noncontractible curves that have length equal to $s y s \pi_{1}$ "systoles".) One consequence of his analysis is that any such extremal metric must be flat at points where exactly two systoles pass through each point in a neighborhood. Furthermore, these systoles must intersect orthogonally.

The study of solutions to the Euler - Lagrange equations for this problem was futher taken up by R. Bryant in [Br96].

Some very interesting examples of piecewise flat (singular) metrics in genus 3 that satisfy these criteria were presented in [Ca96]. They do not have the same 
systolic ratio, one has ratio $\frac{9}{8 \sqrt{3}} \sim .6495$ and the other $\frac{8}{7 \sqrt{3}} \sim .6598$, but Calabi stated in that paper that these were probably both local maxima (or at least stationary points) of the systolic ratio. Today [Ca03] Calabi feels that the example with the smaller systolic ratio (a ramified 4-fold cover of the octahedron with ratio $\frac{9}{8 \sqrt{3}}$ ) is probably not locally a maximum. The example with the larger systolic ratio is still conjectured to be not only a local maximum but the best possible. Calabi [Ca03, Ca3] also has an example on a surface of genus 4 of a piecewise flat (with conical singularities) metric (having a symmetry group of order 120) with a systolic ratio of $\frac{(18 \sqrt{7})^{2}}{1440 \sqrt{7}}=\frac{9 \sqrt{7}}{40} \approx 0.5953$. It is built out of 60 flat rhombi with angles $\arccos (1 / 8)$ and $\arccos (-1 / 8)$. It is suspected that it is close to the optimum systolic ratio but it is not optimum since there are points through which only one systole passes.

An important local solution to the Euler-Lagrange equations for extremal isosystolic metrics, the "Fried Egg", was also described in [Ca96]. This example is a (non flat) metric on a disk whose boundary is a regular hexagon all of whose angles are $\frac{\pi}{2}$. It arises in consideration of the problem of finding the least area Riemannian metric in a hexagon (having the symmetries of a hexagon - i.e. the dihedral group of order 12 generated by reflections) such that each point on a given side has distance exactly 2 to the opposite side. (The above additional symmetry assumption did not appear in the original paper but should be included [Ca03].) A solution to related problems for an octagon and a triangle might be useful in further increasing the systolic ratio in the example of section 2 by replacing the flat octagons (in the dual picture) or the singular triangles with such fried eggs.

The example (piecewise flat with conical singularities) of Calabi above in genus 3 with systolic ratio $\frac{8}{7 \sqrt{3}}$ is the only candidate that exists for metrics on surfaces of higher genus achieving the maximal systolic ratio. In contrast, Sabourau in [Sab02c] shows that in genus 2, no flat metric with conical singularities (such as the example of section 2) can achieve the maximal systolic ratio.

\section{Gromov's Filling Riemannian Manifolds}

In this section, we discuss some of the main results in $[\mathbf{G r 8 3}]$. The reader should look at that paper for many interesting results in this area.

1. Filling radius + main estimate. Let $X^{n}$ be a closed, smooth, $n$-dimensional manifold with a metric $d_{X}$ (not necessarily Riemannian). Let $A$ be a coefficient ring (either $\mathbb{Z}$ or $\mathbb{Z} / 2 \mathbb{Z}$ ). In [Gr83], Gromov introduced the notion of the filling radius, $\operatorname{FillRad}(X, A)$, with respect to $A$, of $\left(X, d_{X}\right)$. There is a natural strong isometric embedding (in the metric space sense!) $i: X \rightarrow L^{\infty} X$, defined by $i(x)(\cdot)=d_{X}(x, \cdot)$. The filling radius is the infimum of $r$ such that $i(X)$ bounds in the tubular $r$-neighborhood

$$
T_{r}(i(X)) \subset L^{\infty} X
$$

in the sense that the image $i([X])$ of the fundamental class vanishes in $H_{n}\left(T_{r}(i(X)) ; A\right)$.

The only Riemannian manifolds for which the precise value of the filling radius is known [Ka83] are spheres and real projective spaces of constant curvature $K$, as well as a single additional case of $\mathbb{C} P^{2}[\mathbf{K a 9 1 B}]$. Thus, FillRad $\left(S^{n}\right)=$ $\frac{1}{2} \arccos \left(-\frac{1}{n+1}\right) K^{-\frac{1}{2}}$. Meanwhile, $\operatorname{FillRad}\left(\mathbb{R} P^{n}\right)=\frac{1}{3} \operatorname{diam}\left(\mathbb{R} P^{n}\right)$, and thus "round" real projective spaces are extremal for the optimal inequality $\operatorname{FillRad}(X) \leq \frac{1}{3} \operatorname{diam}(X)$, 
valid for all Riemannian manifolds [Ka83]. Partial results in the direction of calculating the filling radius for other two-point homogeneous spaces were obtained in [Ka91A, Ka91B, Ka91C]. An optimal inequality for the filling radius appears in [Wi92].

One of the fundamental estimates that allows universal inequalities in higher dimensions is the following theorem, due to Gromov [Gr83, Section 1.2, Main Theorem].

Theorem 3.1. For any closed Riemannian $n$-manifold $X$ we have

$$
\operatorname{vol}(X)^{\frac{1}{n}}>\left((n+1)\left(n^{n}\right) \sqrt{(n+1) !}\right)^{-1} \operatorname{FillRad}(X) .
$$

Many of the higher dimensional universal inequalities use this estimate along with an estimate for the filling radius. The first of these was Gromov's systolic theorem (below) for essential manifolds.

Definition 3.2. The manifold $X^{n}$ is called essential (over $A$ ) if it admits a map, $F: X \rightarrow K$, to an aspherical space $K$ such that the induced homomorphism $F_{*}: H_{n}(X, A) \rightarrow H_{n}(K, A)$ sends the fundamental class [X] to a nonzero class: $F_{*}([X]) \neq 0 \in H_{n}(K, A)$.

Here we must take $A=\mathbb{Z} / 2 \mathbb{Z}$ if $X$ is nonorientable. In [Gr83, Theorem 0.1.A] Gromov showed the following.

Theorem 3.3. Assume $X$ is essential over A. Then every Riemannian metric $\mathbf{g}$ on $X$ satisfies the inequality

$$
\operatorname{FillRad}(\mathbf{g}) \geq \frac{1}{6} s y s \pi_{1}(\mathbf{g})
$$

Combined with Theorem 1 , this yields the inequality

$$
\operatorname{sys}_{1}(\mathbf{g})^{n}<\left(6(n+1)\left(n^{n}\right) \sqrt{(n+1) !}\right)^{n} \operatorname{vol}_{n}(\mathbf{g}) .
$$

This theorem generalizes both the Loewner (2.3) and the $\mathrm{Pu}(2.5)$ theorems to higher dimensions (with non-sharp constants) since both $\mathbb{T}^{n}$ and $\mathbb{R} P^{n}$ are essential, and in particular answers Question 2.11 in the affirmative for these spaces. Since the proof of the theorem is the model for most other estimates of $\operatorname{FillRad}(X)$ we give it now.

Proof. We will represent a filling as continuous map $\sigma: \Sigma \rightarrow T_{r}(i(X))$ from an $(n+1)$-dimensional simplicial complex $\Sigma$ such that the restriction $\left.\sigma\right|_{\partial \Sigma}: \partial \Sigma \rightarrow i(X)$ represents a generator in $H_{n}(X ; A)$. We note that for any fixed $\epsilon>0$, by taking barycentric subdivisions as needed, we may assume that the $\sigma$-image of any simplex has diameter less than $\epsilon$ in $L^{\infty}(X)$. We note that there can be no continuous map $f: \Sigma \rightarrow K$ which agrees with $F \circ \sigma$ on $\partial \Sigma$, since $\left.F \circ \sigma\right|_{\partial \Sigma}$ represents $F_{*}[X]$ (so is not a boundary in $K$ by the hypothesis of Definition 3.2). Since $F$ is defined on $X$, we can also think of it as defined on $i(X) \subset L^{\infty}(X)$ because $i$ is an embedding.

The proof will be by contradiction. We assume that FillRad $(X)<\frac{1}{6} s y s \pi_{1}(X)$, let $0<\epsilon$ be such that 2 FillRad $(X)+3 \epsilon<\frac{1}{3} s y s \pi_{1}(X)$. Take a filling $\sigma: \Sigma \rightarrow$ $T_{r}(i(X)$ ) (for $r=$ FillRad $(X)+\epsilon$ ) where the image of the simplices have diameter 
less than $\epsilon$, and show that $F \circ \sigma: \partial \Sigma \rightarrow K$ extends to a continuous $f: \Sigma \rightarrow K$ giving the desired contradiction.

We construct the extension by first choosing, for every vertex $v$ of $\Sigma-\partial \Sigma$, a point $x(v) \in X$ such that $d(i(x(v)), \sigma(v))<r$ (which we can do since $\sigma(\Sigma) \subset$ $\left.T_{r}(i(X))\right)$. (For $v \in \partial \Sigma$ just take $\left.x(v)=\sigma(v)\right)$ Now we define $f(v) \equiv F(x(v))$. Let $e$ be an edge of a simplex in $\Sigma$ with endpoints $v_{1}$ and $v_{2}$. The strongly isometric nature of the imbedding $X \rightarrow L^{\infty} X$ and the triangle inequality in $L^{\infty} X$ imply that

$$
d\left(x\left(v_{1}\right), x\left(v_{2}\right)\right)=d\left(i\left(x\left(v_{1}\right)\right), i\left(x\left(v_{2}\right)\right)\right) \leq 2 r+\epsilon<\frac{1}{3} s y s \pi_{1}(X) .
$$

Choose a shortest path $x(e)(t)$ from $x\left(v_{1}\right)$ to $x\left(v_{2}\right)$ in $X$ and let $f(e(t))=F(x(e)(t))$. This extends $f$ to the one-skeleton of $\Sigma$. Now for each two-simplex of $\Sigma$ with edges $e_{1}, e_{2}$, and $e_{3}$, the closed curve formed by the $x\left(e_{i}\right)$ has length less than $s y s \pi_{1}(X)$. Therefore it can be contracted to a point in $X$. Hence we can define a map of the two-simplex to $X$, and then composing with $F$ to $K$ which agrees with $f$ on the edges. Thus we can extend $f$ to the two-skeleton. Now since $K$ is aspherical, there is no obstruction to extending $f$ to the rest of $\Sigma$. This gives the desired contradiction.

In general, proofs of lower bounds on the filling radius take this form. That is, one assumes the filling radius is small, takes a nice filling, and uses it to construct some map (usually skeleton by skeleton) that one knows does not exist.

The following theorem from [Gro2, 3. $C_{1}$ ] can be thought of as a generalisation of Loewner's inequality (2.3), and a homological analogue of (3.4), see also Gromov's sharp stable inequality (5.14). Let $X$ be a smooth compact $n$-dimensional manifold. Assume that there is a field $F$ and classes $\alpha_{1}, \ldots, \alpha_{n} \in H^{1}(X, F)$ with a nonvanishing cup product $\alpha_{1} \cup \ldots \cup \alpha_{n} \neq 0$. Then every Riemannian metric $\mathbf{g}$ on $X$ satisfies the inequality

$$
\operatorname{sys}_{1}(\mathbf{g})^{n} \leq C_{n} \operatorname{vol}_{n}(\mathbf{g})
$$

where $C_{n}$ is a constant depending only on the dimension.

In section 4 we introduced the notion of a stationary 1-cycle. These are usually found by the minimax techniques of Almgren and Pitts (see [Al62] and [Pitts81, Theorem 4.10] ). A stationary one cycle is built out of finitely many geodesic segments and the mass is just the sum of the lengths of the segments. For a given Riemannian metric $\mathbf{g}$ we will let $m_{1}(\mathbf{g})$ be the minimal mass of a stationary 1-cycle. Since a closed geodesic is a stationary 1 -cycle we have $L(\mathbf{g}) \geq m_{1}(\mathbf{g})$.

Recently these techniques have been combined with the Filling techniques to get isosystolic type estimates in all dimensions for all manifolds. In [N-R2] Nabutovsky and Rotman showed that

$$
m_{1}(\mathbf{g}) \leq 2(n+2) ! \text { FillRad }(\mathbf{g})
$$

and hence, by Theorem 3.1, we have $m_{1}(\mathbf{g})^{n} \leq C(n) \operatorname{vol}(\mathbf{g})$ (for an explicit constant $C(n))$.

In [Sab1] Sabourau gives a lower bound on the Filling radius of a generic metric on the two-sphere in terms of the minimal mass of a 1-cycle of index 1 . The minimal mass is achieved by either a simple closed geodesic or a figure 8 geodesic. The advantage of this estimate is that short geodesics around thin necks (which, in nearby generic metrics, will have index 0 as 1-cycles) can be ignored to give better bounds on the area. 
2. Filling volume and chordal metrics. In [Gr83] Gromov also introduced the notion of Filling Volume, FillVol $\left(N^{n}, d\right)$, for a compact manifold $N$ with a metric $d$ (here $d$ is a distance function which is not necessarily Riemannian). For the actual definition one should see $[\mathbf{G r 8 3}]$, but it is shown in $[\mathbf{G r 8 3}]$ that when $n \geq 2$

$$
\operatorname{FillVol}\left(N^{n}, d\right)=\inf _{\mathbf{g}} \operatorname{vol}\left(X^{n+1}, \mathbf{g}\right)
$$

where $X$ is any fixed manifold such that $\partial X=N$ (one can even take $X=N \times$ $[0, \infty)$ ), the infimum is taken over all Riemannian metrics $\mathbf{g}$ on $X$ for which the boundary distance function is $\geq d$. In the case $n=1$, the topology of the filling $X^{2}$ could affect the infimum, as is shown by example in [Gr83, Counterexamples 2.2.B].

In fact, the filling volume is not known for any Riemannian metric. However, Gromov does conjecture the following in [Gr83], immediately after Proposition 2.2.A.

Conjecture 3.8. Fillvol $\left(S^{n}\right.$, can $)=\frac{1}{2} \omega_{n+1}$, where $\omega_{n+1}$ represents the volume of the unit $(n+1)$-sphere.

This is still open in all dimensions. In the case that $n=1$, as we pointed out earlier, this is closely related to Conjecture 2.7. The filling area of the circle of length $2 \pi$ with respect to the simply connected filling (by a disk) is indeed $\frac{\pi}{2}$, by $\mathrm{Pu}$ 's inequality (2.5) applied to the projective plane obtained by identifying opposite points of the circle.

In many cases it is more natural to consider "chordal metrics" than Riemannian metrics when discussing Filling volume. Consider a compact manifold $X$ with boundary $\partial X$ with a Riemannian metric $\mathbf{g}$. Then there is a (typically not Riemannian) metric $d_{\mathbf{g}}$ on $\partial X$, where $d_{\mathbf{g}}(x, y)$ represents the distance in $X$ with respect to the the metric $\mathbf{g}$, i.e. the length of the $\mathbf{g}$-shortest path in $X$ between boundary points. We call $d_{\mathbf{g}}$ the chordal metric on $\partial X$ induced by $\mathbf{g}$.

Sharp filling volume estimates for chordal metrics are related to a universal length versus volume question for a given compact manifold $X$ with boundary $\partial X$. This question compares the volumes, $\operatorname{vol}\left(\mathbf{g}_{0}\right)$ and $\operatorname{vol}\left(\mathbf{g}_{1}\right)$, of two Riemannian metrics $\mathbf{g}_{0}$ and $\mathbf{g}_{1}$ on $X$ if we know that for every pair of points $x, y \in \partial X$ we have $d_{\mathbf{g}_{0}}(x, y) \leq d_{\mathbf{g}_{1}}(x, y)$. Of course, without some further assumptions on the metrics (such as some minimizing property of geodesics) there is no general comparison between the volumes. For a fixed $\mathbf{g}_{0}($ and $n \geq 2)$ the statement that for all such $\mathbf{g}_{1}$ we have $\operatorname{vol}\left(\mathbf{g}_{0}\right) \leq \operatorname{vol}\left(\mathbf{g}_{1}\right)$ is just the statement that $F i l l \operatorname{Vol}\left(\partial X, d_{\mathbf{g}_{0}}\right)=\operatorname{vol}\left(\mathbf{g}_{0}\right)$. Note that the standard metric on the $n$-sphere is just the chordal metric of the $(n+1)$-dimensional hemisphere that it bounds. So Conjecture 3.8 is in fact such a question.

In the computation of the Filling volume via formula (3.7) above when $d$ is the chordal distance function of some Riemannian manifold $\left(X^{n+1}, \partial X, \mathbf{g}_{0}\right)(n \geq 2)$ one can not only fix the topology of $X^{n+1}$ but also restrict to metrics $\mathbf{g}$ such that the Riemannian metrics on $\partial X$ gotten by restricting $\mathbf{g}$ and $\mathbf{g}_{0}$ to $\partial X$ are the same (see [Cr01]).

The filling volume is known for some chordal metrics. Gromov in [Gr83] proved this for $X^{n+1}$ a compact subdomain of $\mathbb{R}^{n+1}$ (or in fact for some more general flat manifolds with boundary). The minimal entropy theorem of Besson, Courtois, and Gallot [B-C-G96, B-C-G95] can be used to prove the result for compact 
subdomains of symmetric spaces of negative curvature (see [Cr01]). For general convex simply connected manifolds $\left(X, \partial X, \mathbf{g}_{0}\right)$ of negative curvature, there is a $C^{3}$ neighborhood in the space of metrics such that any metric $\mathbf{g}_{1}$ in that neighborhood with $\left.\mathbf{g}_{1}\right|_{\partial X}=\left.\mathbf{g}_{0}\right|_{\partial X}$ and $d_{\mathbf{g}_{1}}(x, y) \geq d_{\mathbf{g}_{0}}(x, y)$ has $\operatorname{vol}\left(\mathbf{g}_{1}\right) \geq \operatorname{vol}\left(\mathbf{g}_{0}\right)$ and equality of the volumes implies $\mathbf{g}_{1}$ is isometric to $\mathbf{g}_{0}$ (see [C-D-S00]). This leads to the conjecture:

Conjecture 3.9. For any compact subdomain of a simply connected space of negative (nonpositive?) curvature of dimension $\geq 3$, the filling volume of the boundary with the chordal metric is just the volume of the domain. Furthermore, the domain is the unique (up to isometry) volume minimizing filling.

The uniqueness part of this question has applications to the boundary rigidity problem. In that problem one considers the case when $d_{\mathbf{g}_{0}}(x, y)=d_{\mathbf{g}_{1}}(x, y)$ for all boundary points $x$ and $y$ and asks if $\mathbf{g}_{0}$ must be isometric to $\mathbf{g}_{1}$. In some natural cases (see $S G M$ below) the volumes can be shown to be equal. For example, Gromov's result for subdomains of Euclidean space showed that they were boundary rigid. Again this cannot hold in general. A survey of what is known about the boundary rigidity problem can be found in [Cr01]. There are a few natural choices for assumptions in this case. The most general natural assumption of this sort is $S G M$. The $S G M$ condition (which is given in terms of the distance function $d_{\mathbf{g}}: \partial X \times \partial X \rightarrow \mathbb{R}$ alone) would take some space to define precisely (see [Cr91]), but loosely speaking (i.e. the definitions coincide except in a few cases) it means the following:

Definition 3.10. A metric is "loosely" $S G M$ if all nongrazing geodesic segments are strongly minimizing.

By a nongrazing geodesic segment we mean a segment of a geodesic which lies in the interior of $X$ except possibly for the endpoints. A segment is said to minimize if its length is the distance between the endpoints, and to strongly minimize if it is the unique such path. (This loose definition seems to rely on more than $d_{\mathbf{g}}$ but the relationship is worked out in [Cr91].) Examples of such $(X, \partial X, \mathbf{g})$ are given by compact subdomains of an open ball, $B$, in a Riemannian manifold where all geodesics segments in $B$ minimize. The only reason not to use the "loose" definition above is that using a definition (such as $S G M$ ) given in terms only of $d_{\mathbf{g}}$ guarantees that if $d_{0}(x, y)=d_{1}(x, y)$ and $\mathbf{g}_{0}$ is $S G M$, then $\mathbf{g}_{1}$ will be as well. In fact, the questions and results stated here for the $S G M$ case also hold for manifolds satisfying the loose definition, so the reader can treat that as a definition of $S G M$ for the purpose of this paper.

The most general result one could hope for would be of the form:

Question 3.11. If $\mathbf{g}_{0}$ is an $S G M$ metric on $(X, \partial X)$ and $\mathbf{g}_{1}$ is another metric with $d_{\mathbf{g}_{0}}(x, y) \leq d_{\mathbf{g}_{1}}(x, y)$ for $x, y \in \partial X$ then $\operatorname{vol}\left(\mathbf{g}_{0}\right) \leq \operatorname{vol}\left(\mathbf{g}_{1}\right)$ with equality of volumes implying $\mathbf{g}_{0}$ is isometric to $\mathbf{g}_{1}$.

This is still very much an open question, which as stated includes the boundary rigidity problem. 
The case $\mathbf{g}_{1}=f^{2}(x) \mathbf{g}_{0}$ (i.e. $\mathbf{g}_{1}$ is pointwise conformal to $\mathbf{g}_{0}$ ) was answered positively in [C-D] (also see [Cr91]).

In two dimensions more is known. Recently Ivanov [Iv02] considered the case of compact metrics $\mathbf{g}_{0}$ and $\mathbf{g}_{1}$ on a disk. He assumes that $\mathbf{g}_{0}$ is a convex metric in the sense that every pair of interior points can be joined by a unique geodesic, and proves that if $d_{\mathbf{g}_{1}} \geq d_{\mathbf{g}_{0}}$ then $\operatorname{vol}\left(\mathbf{g}_{1}\right) \geq \operatorname{vol}\left(\mathbf{g}_{0}\right)$. He also says that equality in the area would imply that $d_{\mathbf{g}_{1}}=d_{\mathbf{g}_{0}}$. Of course, going from here to isometry of $\mathbf{g}_{0}$ and $\mathbf{g}_{1}$ is the boundary rigidity problem. This boundary rigidity problem (in 2 dimensions) is solved $[\mathbf{C r 9 1}, \mathbf{O t 9 0 B}]$ in the case that the metric $\mathbf{g}_{0}$ has negative curvature.

Using different methods [C-D] proves a similar though somewhat different result where the metrics $\mathbf{g}_{0}$ and $\mathbf{g}_{1}$ are both assumed to be $S G M$ but the surfaces are not assumed to be simply connected. The estimate comes from a formula for the difference between the areas of two $S G M$ surfaces. Since the surfaces are not simply connected one needs to worry about the homotopy class of a path between two boundary points. We will let $L_{\mathbf{g}}(x, y,[\alpha])$ be the length of the g-shortest curve from $x$ to $y$ in a homotopy class $[\alpha]$ of curves from $x$ to $y$. $\mathcal{A}$ will represent the space of such triples $(x, y,[\alpha])$ The formula is:

$$
\operatorname{area}\left(\mathbf{g}_{1}\right)-\operatorname{area}\left(\mathbf{g}_{0}\right)=\frac{1}{2 \pi} \int_{\mathcal{A}}\left(L_{\mathbf{g}_{1}}(x, y,[\alpha])-L_{\mathbf{g}_{0}}(x, y,[\alpha])\right)\left(\mu_{\mathbf{g}_{1}}+\mu_{\mathbf{g}_{0}}\right),
$$

where the measures $\mu_{\mathbf{g}_{i}}$ on $\mathcal{A}$ are the push forwards of the standard Liouville measure on the space of geodesics. Since for each pair $(x, y)$ there is at most one geodesic segment in each metric from $x$ to $y$, we see that for most $(x, y,[\alpha])$ there will be neither a $\mathbf{g}_{1}$-geodesic nor a $\mathbf{g}_{2}$-geodesic segment from $x$ to $y$ in the class $[\alpha]$ and hence the term for $(x, y,[\alpha])$ will contribute nothing to the integral (see $[\mathbf{C}-\mathbf{D}]$ for details). This formula easily leads to a result relating lengths of paths to area.

Another powerful result with filling volume consequences is the Besicovitch lemma which was exploited and generalized in [Gr83, section 7]. It says that for any Riemannian metric on a cube, the volume is bounded below by the product of the distances between opposite faces. Equality only holds for the Euclidean cube.

3. Marked length spectrum and volume. The question for compact manifolds $N$ without boundary corresponding to Question 3.11 involves the marked length spectrum. The marked length spectrum for a Riemannian metric $\mathbf{g}$ on $N$ is a function, $M L S_{\mathbf{g}}: \mathcal{C} \rightarrow \mathbb{R}^{+}$, from the set $\mathcal{C}$ of free homotopy classes of the fundamental group $\pi_{1}(N)$ to the nonnegative reals. For each $\langle\gamma\rangle \in \mathcal{C}, M L S_{\mathbf{g}}(\langle\gamma\rangle)$ is the length of the shortest curve in $\langle\gamma\rangle$ (always a geodesic). We consider two Riemannian metrics $\mathbf{g}_{0}$ and $\mathbf{g}_{1}$ on $N$ such that $M L S_{\mathbf{g}_{1}}(\langle\gamma\rangle) \geq M L S_{\mathbf{g}_{0}}(\langle\gamma\rangle)$ for all free homotopy classes $\langle\gamma\rangle$ (we then say $\left.M L S_{\mathbf{g}_{1}} \geq M L S_{\mathbf{g}_{0}}\right)$ and ask if $\operatorname{vol}\left(\mathbf{g}_{1}\right)$ must be greater than or equal to $\operatorname{vol}\left(\mathbf{g}_{0}\right)$. Again this is hopeless without further assumptions.

The natural setting for this is in negative curvature. Here there are lots of closed geodesics but exactly one for each free homotopy class (achieving the minimum length in that class). The following was conjectured in [C-D-S00]:

Conjecture 3.13. For two negatively curved metrics, $\mathbf{g}_{0}$ and $\mathbf{g}_{1}$, on a manifold $N$ the inequality $M L S_{\mathbf{g}_{1}} \geq M L S_{\mathbf{g}_{0}}$ implies $\operatorname{vol}\left(\mathbf{g}_{1}\right) \geq \operatorname{vol}\left(\mathbf{g}_{0}\right)$. Furthermore, $\operatorname{vol}\left(\mathbf{g}_{1}\right)=$ $\operatorname{vol}\left(\mathbf{g}_{0}\right)$ if and only if $\mathbf{g}_{0}$ and $\mathbf{g}_{1}$ are isometric. 
This conjecture was proved in dimension 2 in [C-D]. The higher dimensional version of the above was shown when $\mathbf{g}_{0}$ and $\mathbf{g}_{1}$ are pointwise conformal. This used ideas developed in [Bo91] and [Si90].

A consequence of this conjecture is the conjecture for negatively curved $\mathbf{g}_{0}$ and $\mathbf{g}_{1}$ that $M L S_{\mathbf{g}_{1}}=M L S_{\mathbf{g}_{0}}$ would imply $\mathbf{g}_{0}$ is isometric to $\mathbf{g}_{1}$. See [Cr01] for a survey of this problem and [Ban94, C-F-F92, Cr91, Ot90A] for results (stronger than the conjecture) in two dimensions. However, it is not even known if $M L S_{\mathbf{g}_{1}}=M L S_{\mathbf{g}_{0}}$ implies $\operatorname{vol}\left(\mathbf{g}_{1}\right)=\operatorname{vol}\left(\mathbf{g}_{0}\right)$. In fact this is itself an important open question. Hamenstädt [Ha99] (also see [Ha92]) proved this in the special case that $\mathbf{g}_{0}$ is further assumed to be locally symmetric. This was the case that had the most important immediate applications. For example, it allows one to drop the volume assumption in the conjugacy rigidity result in [B-C-G95] and hence to see that if $\mathbf{g}_{0}$ is locally symmetric and $\mathbf{g}_{1}$ is a metric of negative curvature with $M L S_{\mathbf{g}_{1}}=M L S_{\mathbf{g}_{0}}$, then $\mathbf{g}_{0}$ is isometric to $\mathbf{g}_{1}$.

\section{Systolic freedom for unstable systoles}

In [Gr83, p. 5], M. Gromov, following M. Berger, asks the following basic question. What is the best constant $C$, possibly depending on the topological type of the manifold, for which the $k$-systolic inequality (4.2) holds? It was shown by the second author in collaboration with A. Suciu [KS99, KS01] that such a constant typically does not exist whenever $k>1$, in sharp contrast with the inequalities of Loewner, $\mathrm{Pu}$, and Gromov discussed in sections 2 and 3.

Similar non-existence results were obtained for a pair of complementary dimensions (4.4), culminating in the work of I. Babenko [Bab02], cf. section 4. These results are placed in the context of other systolic results in the table of Figure 4.1.

1. Table of systolic results, definitions. Let $(X, \mathbf{g})$ be a Riemannian manifold, and let $k \in \mathbb{N}$. Given a homology class $\alpha \in H_{1}(X ; A)$, denote by $\operatorname{len}(\alpha)$ the least length (in the metric $\mathbf{g}$ ) of a 1-cycle with coefficients in $A$ representing $\alpha$. The homology 1-systole sys ${ }_{1}(\mathrm{~g}, A)$ is defined by setting

$$
\operatorname{sys}_{1}(\mathbf{g}, A)=\min _{\alpha \neq 0 \in H_{1}(X, A)} \operatorname{len}(\alpha),
$$

and we let sys $_{1}(\mathbf{g})=$ sys $_{1}(\mathrm{~g}, \mathbb{Z})$. The other important case is $s y s_{1}\left(\mathbf{g}, \mathbb{Z}_{2}\right)$. In other words, sys $s_{1}(\mathbf{g})$ is the length of a shortest loop which is not nullhomologous in a compact Riemannian manifold $(X, \mathbf{g})$ with a nontrivial group $H_{1}(X, \mathbb{Z})$.

More generally, let $\operatorname{sys}_{k}(\mathbf{g})$ be the infimum of $k$-volumes of integer (Lipschitz) $k$ cycles which are not boundaries in $X, c f$. formula (4.1). Note that if $X$ is orientable of dimension $n$, then the total volume is a systolic invariant: $\operatorname{vol}_{n}(\mathbf{g})=\operatorname{sys}_{n}(\mathbf{g})$.

The term "systolic freedom" refers to the absence of a systolic inequality, e.g. violation of the inequality relating a single systole to the total volume, namely

$$
\operatorname{sys}_{k}(\mathbf{g})^{\frac{n}{k}}<\operatorname{Cvol}_{n}(\mathbf{g}),
$$

or similarly the inequality involving a pair of complementary dimensions, $k$ and $(n-k)$, namely $\operatorname{sys}_{k}(\mathbf{g}) s y s_{n-k}(\mathbf{g})<\operatorname{Cvol}_{n}(\mathbf{g})$, by a suitable sequence of metrics, $c f$. formula (4.5). Thus, we say that an $n$-manifold is $k$-systolically free if

$$
\inf _{\mathbf{g}} \frac{\operatorname{vol}_{n}(\mathbf{g})}{\operatorname{sys}_{k}(\mathbf{g})^{n / k}}=0
$$




\begin{tabular}{|c|c|c|}
\hline & $1 \quad k=1$ & $2 k \geq 2$ \\
\hline $\begin{array}{l}\mathrm{A} \\
\text { homotopy } \\
k \text {-systole }\end{array}$ & $\begin{array}{l}\text { Loewner's inequality for } \mathbb{T}^{2}(2.3) \text {, } \\
\text { Pu's for } \mathbb{R} P^{2}(2.5), \text { Gromov's for } \\
\text { essential } X(3.4)\end{array}$ & \\
\hline $\begin{array}{l}\mathrm{B} \\
\text { homology } \\
k \text {-systole }\end{array}$ & $\begin{array}{l}\text { if cuplength }\left(X^{n}\right)=n \text { then Gro- } \\
\text { mov proves inequality }(3.5)\end{array}$ & $\begin{array}{l}\text { freedom reigns: Gromov (4.5), } \\
\text { Babenko (4.3), (4.4); Katz, Suciu (4.3); } \\
\text { freedom of } S^{1} \times S^{2} \text { over } \mathbb{Z}_{2} \text { : Freedman } \\
(4.6)\end{array}$ \\
\hline $\begin{array}{l}\mathrm{C} \\
\text { stable homol- } \\
\text { ogy } k \text {-systole }\end{array}$ & $\begin{array}{l}\text { sharp inequality if } b_{1}\left(X^{n}\right)=\text { cup- } \\
\text { length }\left(X^{n}\right)=n \text { (Gromov, based } \\
\text { on Burago-Ivanov) }(5.14)\end{array}$ & $\begin{array}{l}\text { multiplicative relations in } H^{*}(X) \text { entail } \\
\text { inequalities: Gromov, Hebda (5.15), } \\
\text { Bangert-Katz (5.13), (5.17) }\end{array}$ \\
\hline $\begin{array}{l}\mathrm{D} \\
\text { interpolation } \\
\text { homotopy- } \\
\text { homology }\end{array}$ & $\begin{array}{l}\text { "fiberwise" inequality if Abel- } \\
\text { Jacobi map "surjective" (Katz- } \\
\text { Kreck-Suciu) [KKS], cf. (5.12) }\end{array}$ & \\
\hline $\begin{array}{l}\mathrm{E} \\
\text { conformal } \\
k \text {-systole }\end{array}$ & $\begin{array}{l}\text { Accola, Blatter (section 3); for } \\
\text { a surface } \Sigma_{s}, \quad \text { logarithmic in } \\
\text { genus } s \text { : Buser-Sarnak }(5.8)\end{array}$ & $\begin{array}{l}X^{4} \text {, indefinite case: polynomial in } \\
\chi(X) \text {, modulo surjectivity of period } \\
\text { map: Katz }(5.10)\end{array}$ \\
\hline $\begin{array}{l}\mathrm{F} \\
\text { contractible } \\
k \text {-systole }\end{array}$ & \begin{tabular}{llll} 
short & \multicolumn{2}{c}{ contractible geodesics: } \\
Croke $(2.13)$, & Maeda, & Nab- \\
utovsky and Rotman & $(3.6)$, \\
Sabourau $(6.5)$ & &
\end{tabular} & \\
\hline
\end{tabular}

Figure 4.1. A 2-D map of systolic geometry

and $(k, n-k)$-systolically free if

$$
\inf _{\mathbf{g}} \frac{\operatorname{vol}_{n}(\mathbf{g})}{\operatorname{sys}_{k}(\mathbf{g}) s y s_{n-k}(\mathbf{g})}=0
$$

where the infimum is over all smooth metrics $\mathrm{g}$ on the manifold. See Section 4 for results in this direction. Note that we use the reciprocals of our convention (2.2) for the systolic ratio, when discussing systolic freedom.

2. Gromov's homogeneous (1,3)-freedom. M. Gromov first described a $(1,3)$-systolically free family of metrics on $S^{1} \times S^{3}$ in 1993, cf. [Gr96, section 4.A.3], [Gr99, p. 268]. His construction of (1,3)-systolic freedom on the manifold $S^{1} \times S^{3}$ exhibits a family $\mathbf{g}_{\epsilon}$ of homogeneous metrics with the following asymptotic behavior:

$$
\frac{\operatorname{vol}_{4}\left(\mathbf{g}_{\epsilon}\right)}{\operatorname{sys}_{1}\left(\mathbf{g}_{\epsilon}\right) \text { sys }_{3}\left(\mathbf{g}_{\epsilon}\right)} \rightarrow 0 \text { as } \epsilon \rightarrow 0 .
$$

Due to the exceptional importance of this example, we present a proof. Let $U(2)$ be the unitary group acting on the unit sphere $S^{3} \subset \mathbb{C}^{2}$. In particular, we have the scalar matrices $e^{i \theta} I_{2} \in U(2)$. Consider the direct sum metric on $\mathbb{R} \times S^{3}$, and let $\alpha$ be the pullback to $\mathbb{R} \times S^{3}$ of the volume form of the second factor. Let Trans $(\mathbb{R})$ be the group of translations of the real line. Let $\epsilon>0$ be a real parameter. We will define an infinite cyclic subgroup $\Gamma_{\epsilon} \subset \operatorname{Trans}(\mathbb{R}) \times U(2)$ of the group of isometries of $\mathbb{R} \times S^{3}$, in such a way that the quotient

$$
\left(\mathbb{R} \times S^{3}\right) / \Gamma_{\epsilon} \simeq\left(S^{1} \times S^{3}, \mathbf{g}_{\epsilon}\right)
$$


produces a $(1,3)$-systolically free family of metrics $\mathbf{g}_{\epsilon}$ on $S^{1} \times S^{3}$ as $\epsilon \rightarrow 0$. Namely, we may take the generator $\gamma_{\epsilon} \in \Gamma_{\epsilon}$ to be the element $\gamma_{\epsilon}=\left(\epsilon, e^{i \sqrt{\epsilon}} I_{2}\right) \in \operatorname{Trans}(\mathbb{R}) \times$ $U(2)$. Then the quantity $\operatorname{sys}_{1}\left(\mathbf{g}_{\epsilon}\right)$ is dominated by the second factor $e^{i \sqrt{\epsilon}}$, and is asymptotic to $\sqrt{\epsilon}$. Meanwhile, sys $s_{3}\left(\mathbf{g}_{\epsilon}\right)=\omega_{3}$, where $\omega_{3}$ the volume of the unit 3 -sphere, by a calibration argument. Namely, every nontrivial 3-cycle $C$ in $S^{1} \times S^{3}$ satisfies $\operatorname{vol}_{3}(C) \geq \int_{C} \alpha=n \omega_{3} \geq \omega_{3}$, where $n$ is the order of $[C] \in H_{3}\left(S^{1} \times S^{3}\right)=\mathbb{Z}$.

Note that we have a fundamental domain with closure $[0, \epsilon] \times S^{3} \subset \mathbb{R} \times S^{3}$ in the universal cover. Hence

$$
\frac{\operatorname{vol}_{4}\left(\mathbf{g}_{\epsilon}\right)}{\operatorname{sys}_{1}\left(\mathbf{g}_{\epsilon}\right) \operatorname{sys}_{3}\left(\mathbf{g}_{\epsilon}\right)} \sim \frac{\epsilon \omega_{3}}{\sqrt{\epsilon} \omega_{3}}=\sqrt{\epsilon} \rightarrow 0
$$

proving (1,3)-systolic freedom of $S^{1} \times S^{3}$.

Note that a calculation of the stable 1-systole, $c f$. formula (5.5), reveals the behavior of systolic constraint instead of freedom. Thus, the $n$-th power of the generator $\gamma_{\epsilon} \in \pi_{1}\left(S^{1} \times S^{3}\right)=\Gamma_{\epsilon}$, where $n=\left[\frac{2 \pi}{\sqrt{\epsilon}}\right]$ is the integer part, also contains a closed geodesic whose length is on the order of $2 \pi \sqrt{\epsilon}$, as

$$
\gamma_{\epsilon}^{n}=\left(n \epsilon, e^{i \sqrt{\epsilon} n} I_{2}\right) \sim\left(2 \pi \epsilon^{-\frac{1}{2}} \epsilon, e^{2 \pi i} I_{2}\right)=\left(2 \pi \epsilon^{\frac{1}{2}}, I_{2}\right) .
$$

It follows that the stable norm satisfies

$$
\left\|\gamma_{\epsilon}\right\| \sim n^{-1} \text { length }\left(\gamma_{\epsilon}^{n}\right) \sim \frac{2 \pi \sqrt{\epsilon}}{n} \sim \epsilon
$$

resulting in a stable 1-systole on the order of $\epsilon$ instead of $\sqrt{\epsilon}$, which is consistent with Hebda's inequality (5.15).

Remarkably, M. Freedman [Fr99] proves the (1,2)-systolic freedom of $S^{1} \times S^{2}$ even when one works with homology with coefficients in $\mathbb{Z}_{2}$, i.e. nonorientable surfaces are allowed to compete in the definition of the 2 -systole. He showed that

$$
\inf _{\mathbf{g}} \frac{\operatorname{vol}_{3}(\mathbf{g})}{\text { sys }_{1}\left(\mathbf{g}, \mathbb{Z}_{2}\right) \operatorname{sys}_{2}\left(\mathbf{g}, \mathbb{Z}_{2}\right)}=0
$$

where the infimum is over all metrics $\left(S^{1} \times S^{2}, \mathbf{g}\right), c f$. formula (4.1). His technique relies on a common starting point with the lower bound of the Buser-Sarnak Theorem [BuSa94] ( $c f .(5.8)$ ), namely the existence of Riemann surfaces of arbitrarily high genus with a uniform lower bound for the positive eigenvalues of the Laplacian. Whether or not $\left(\mathbb{R} P^{3}, \mathbf{g}\right)$ satisfies $(4.6)$ is unknown.

3. Pulling back freedom by $(n, k)$-morphisms. The early papers in the subject [Ka95, Pitte97] contain explicit constructions of systolically free metrics, using geometrically controlled surgery. Here parts of the manifold $X^{n}$ look like a lower dimensional manifold

$$
M^{m}, \text { where } m \leq n-1,
$$

or more concretely, like circle bundle over such an $M$, with short circle fibers. In particular, such constructions yield free families of metrics on products of spheres.

Later, [BabK98] developed a more general framework for constructing metrics well adapted for the systolic problem. This was done in the context of simplicial maps $f$, where some of the top dimensional simplices are collapsed to walls of positive codimension. A positive quadratic form is obtained by "pullback" by $f$, suitably interpreted. The form can then be inflated a little to make it definite, yielding a true smooth metric. This can be thought of as a generalisation of the 
short circle fiber construction, $c f$. (4.7). The next step was to realize that metrics can be pulled back by an even more general morphism, defined below.

Let $X$ and $Y$ be $n$-dimensional simplicial complexes. Notions of volume, as well as systole with coefficients in $A$, can be defined for such objects by using piecewise smooth metrics.

A morphism $_{n, k}$ "from $X$ to $Y$ " (over $A$ ) is a continuous map $f: X \rightarrow W$ satisfying the following two conditions:

(a) the simplicial complex $W$ is obtained from the "target" $Y$ by attaching cells of dimension at most $n-1$;

(b) the map $f$ induces a monomorphism in $k$-dimensional homology, $H_{k}(X, A) \hookrightarrow$ $H_{k}(W, A)$.

Note that the positive codimension of the attached cells parallels the positive codimension in (4.7) above.

Morphisms of this type were referred to as "topological meromorphic maps" in [KS99]. Such a notion encompasses on the one hand, surgeries (cf. item 2 of subsection 4), and on the other, complex blow ups, in suitable contexts. The importance of these morphisms $_{n, k}$ stems from the following observation [BabK98, KS99].

Proposition 4.8. If $X$ admits a morphism ${ }_{n, k}$ to $Y$, then the $k$-systolic freedom of $X$ follows from that of $Y$.

In the problem of systolic freedom, the appropriate objects are not manifolds but CW-complexes $X$, even though piecewise smooth metrics can only be defined on simplicial complexes $X^{\prime}$ homotopy equivalent to $X$. To establish the $k$-systolic freedom for a general $X$, one first maps $X$, by a map inducing a monomorphism on $H_{k}(X, \mathbb{R})$, to a product of $b_{k}(X)$ copies of the loop space $\Omega\left(S^{k+1}\right)$. The space $\Omega\left(S^{k+1}\right)$ is of the rational homotopy type of the Eilenberg Maclane space $K(\mathbb{Z}, k)$.

One uses the existence of a cell structure, due to Morse, which is sparse (in the sense that only cells in an arithmetic progression of dimensions occur) in the homotopy type of $\Omega\left(S^{k+1}\right)$. This reduces the problem to a single $\Omega\left(S^{k+1}\right)$, rather than a Cartesian product, and thus eliminates the dependence on the Betti number. The $k$-systolic freedom of the skeleta of the latter is established by finding a morphism $_{n, k}$ to a product of $k$-spheres, $c f$. Proposition 4.8 .

This is done by analyzing the cell structure of a suitable rationalisation, where the cases $k=2$ and $k \geq 4$ must be treated separately ( $k$ odd is easy). In the former case, one relies on the telescope model of Sullivan, and in the latter, on the existence of rational models with cells of dimension at most $n-k+2$ added (where $n=\operatorname{dim} X$ ) in the construction of the morphism $_{n, k}, c f$. Proposition 4.8 and [KS99, KS01] for details.

4. Systolic freedom of complex projective plane, general freedom. The 2-systolic freedom of $\mathbb{C} P^{2}[\mathbf{K S 9 9}]$ answers in the negative the question from [Gr83, p. 136]. Such freedom results through the following 4 steps.

1. The space $\mathbb{C} P^{2}$ admits a morphism $m_{4,2}$ to $S^{2} \times S^{2}$. Here a 3-cell is attached along a diagonal class in $\pi_{2}\left(S^{2} \times S^{2}\right)$. 
2. The space $S^{2} \times S^{2}$ admits a morphism $m_{4,2}$ to $S^{1} \times S^{1} \times S^{2}$. Here a pair of 2-cells is attached along the generators of $\pi_{1}\left(S^{1} \times S^{1} \times S^{2}\right)$, corresponding to a surgery that allows one to pass from $S^{1} \times S^{1} \times S^{2}$ to $S^{2} \times S^{2}$.

3. There exists a $(1,2)$-systolically free sequence of metrics $\mathbf{g}_{j}$ on the 3 -manifold $S^{1} \times S^{2}$ (cf. [Ka95, Pitte97] and formula (4.5) above).

4. The 2-systolic freedom of $S^{1} \times S^{1} \times S^{2}$ results from the Kunneth formula, by taking Cartesian product of $\mathbf{g}_{j}$ with a circle $S^{1}$ of length sys $_{2}\left(\mathbf{g}_{j}\right) /$ sys $_{1}\left(\mathbf{g}_{j}\right)$.

Part of the procedure described above can be carried out with torsion coefficients. Starting with Freedman's metrics (4.6) on $S^{1} \times S^{2}$, we can construct in this way 2-systolically free metrics on $S^{2} \times S^{2}$ even in the sense of $\mathbb{Z}_{2}$ coefficients. However, the 2-systolic freedom of $\mathbb{C} P^{2}$ with $\mathbb{Z}_{2}$ coefficients is still open. The technique described above fails over $\mathbb{Z}_{2}$ because the map in step 1 has even degree, violates

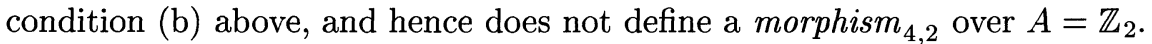

The series of papers [Ka95, Pitte97, BabK98, BKS98, KS99, KS01, Bab02] proved general $k$-systolic freedom, as well as freedom in a pair of complementary dimensions, as soon as any systole other than the first one is involved. Thus, every closed $n$-manifold is $k$-systolically free in the sense of formula (4.3) whenever $2 \leq k<n$ and $H_{k}(X, \mathbb{Z})$ is torsionfree [KS99, KS01]. This answers the basic question of [Gr83, p. 5]. Similarly, every $n$-dimensional polyhedron is $(k, n-k)$-systolically free in the sense of formula (4.4) whenever $1 \leq k<n-k<n$ and $H_{n-k}(X, \mathbb{Z})$ is torsionfree [Bab02]. Simultaneous $(k, n-k)$-systolic freedom for a pair of adjacent values of $k$ is explored in [Ka02].

\section{Stable systolic and conformal inequalities}

M. Gromov showed in $[\mathbf{G r 8 3}, 7.4 . \mathrm{C}]$ that to multiplicative relations in the cohomology ring of a manifold $X$, are associated stable systolic inequalities, satisfied by an arbitrary metric on $X, c f$. (5.13). These inequalities follow from the analogous, stronger inequalities for conformal systoles. He points out that the dependence of the constants in these inequalities on the Betti numbers is unsatisfactory. The invariants involved are defined in (5.5) and (5.7). We first point out the following open question.

Conjecture 5.1. Every closed orientable smooth 4-manifold $X$ satisfies the inequality

$$
\operatorname{stsys}_{2}(\mathbf{g})^{2} \leq \mathrm{Cvol}_{4}(\mathbf{g}), \forall \mathbf{g}
$$

for a suitable numerical constant $C$ (independent of $X$ ).

The lower bound of (5.10) shows that one is unlikely to prove such an inequality via conformal systoles, except in the case of the connected sum of copies of $\mathbb{C} P^{2}$, when (5.2) holds with $C=6, c f$. [Ka3]. The best one has available in general is $C=6 b_{2}(X)[$ BanK03].

Let $H_{1}(X ; \mathbb{Z})_{\mathbb{R}}$ denote the lattice (i.e. free $\mathbb{Z}$-module) obtained as the quotient of $H_{1}(X ; \mathbb{Z})$ by its torsion subgroup. The function $i \mapsto \operatorname{len}(i \alpha)$ defines a norm \|I $\|$, called stable norm $[\mathbf{F e 7 4}, 4.10],[\mathbf{G r 9 9}, 4.35]$, on $H_{1}(X ; \mathbb{Z})_{\mathbb{R}}$ by setting

$$
\left\|\alpha_{\mathbb{R}}\right\|=\lim _{i \rightarrow \infty} i^{-1} \operatorname{len}(i \alpha) \text {. }
$$

Denote by $\lambda_{1}(L,\|\|)$ the least length of a nonzero vector of a lattice $L$ with respect to a norm \|\| . More generally, let $i$ be an integer satisfying $1 \leq i \leq r k(L)$. The 
$i$-th successive minimum $\lambda_{i}(L,\|\|)$ is the least $\lambda>0$ such that there exist $i$ linearly independent vectors in $L$ of norm at most $\lambda$ :

$$
\lambda_{i}(L,\|\|)=\inf _{\lambda}\left\{\lambda \in \mathbb{R} \mid \exists v_{1}, \ldots, v_{i}(l . i .) \forall k=1, \ldots, i:\left\|v_{k}\right\| \leq \lambda\right\}
$$

The stable homology $k$-systole, denoted $\operatorname{stsys}_{k}(\mathbf{g})$, is the least norm of a nonzero element in the lattice $H_{k}(X ; \mathbb{Z})_{\mathbb{R}}$ with respect to the stable norm \| \|:

$$
\text { stsys }_{k}(\mathbf{g})=\lambda_{1}\left(H_{k}(X ; \mathbb{Z})_{\mathbb{R}},\|\|\right) \text {. }
$$

It can be shown that if $(X, \mathbf{g})$ is an orientable surface then $\operatorname{sys}_{1}(\mathbf{g}, \mathbb{Z})=$ stsys $_{1}(\mathbf{g})$.

The conformally invariant norm \|\|$_{L^{n}}$ in $H_{1}\left(X^{n}, \mathbb{R}\right)$ is by definition dual to the conformally invariant $L^{n}$-norm in de Rham cohomology. The latter norm on $H^{1}(X, \mathbb{R})$ is the quotient norm of the corresponding norm on closed forms. Thus, given $\alpha \in H^{1}(X, \mathbb{R})$, we set

$$
\|\alpha\|_{L^{n}}=\inf _{\omega}\left\{\left(\int_{X}\left|\omega_{x}\right|^{n} d \operatorname{vol}(x)\right)^{\frac{1}{n}} \mid \omega \in \alpha\right\},
$$

where $\omega$ runs over closed one-forms representing $\alpha$. On a surface, or more generally for a middle-dimensional class $\alpha \in H^{p}(X, \mathbb{R})$ where $\operatorname{dim}(X)=2 p$, we may write

$$
\|\alpha\|_{L^{2}}^{2}=\int_{X} \omega \wedge * \omega
$$

where $\omega$ is the harmonic representative of $\alpha$ and $*$ is the Hodge star operator of the metric $\mathbf{g}$. The conformal 1-systole of $\left(X^{n}, \mathbf{g}\right)$ is the quantity

$$
\operatorname{confsys}_{1}(\mathbf{g})=\lambda_{1}\left(H_{1}(X, \mathbb{Z})_{\mathbb{R}},\|\|_{L^{n}}\right),
$$

satisfying stsys $_{1}(\mathbf{g}) \leq$ confsys $_{1}(\mathbf{g}) \operatorname{vol}(\mathbf{g})^{\frac{1}{n}}$ on an $n$-manifold $(X, \mathbf{g})$.

1. Asymptotic behavior of conformal systole as function of $\chi(X)$. The inequality of Accola and Blatter is valid for the conformal systole. Their bound was improved by P. Buser and P. Sarnak [BuSa94], who proved that if $\Sigma_{s}$ is a closed orientable surface of genus $s$, the conformal 1-systole satisfies the bounds

$$
C^{-1} \log s<\sup _{\mathbf{g}}\left\{\lambda_{1}\left(H^{1}\left(\Sigma_{s}, \mathbb{Z}\right),\left.\right|_{L^{2}}\right)\right\}^{2}<C \log s, \forall s=2,3, \ldots
$$

where $C>0$ is a numerical constant, the supremum is over all conformal structures g on $\Sigma_{s}$, and ||$_{L^{2}}$ is the associated $L^{2}$-norm, $c f$. formula (5.6). An explicit upper bound of $\frac{3}{\pi} \log (4 s+3)$ in (5.8) is provided in [BuSa94, formula (1.13)]. Note that by Poincaré duality, $\lambda_{1}\left(H^{1}\left(\Sigma_{s}, \mathbb{Z}\right),||_{L^{2}}\right)=\lambda_{1}\left(H_{1}\left(\Sigma_{s}, \mathbb{Z}\right),||_{L^{2}}\right)$.

It should be kept in mind that the asymptotic behavior of the 1-systole as a function of the genus is completely different from the conformal length. Indeed, M. Gromov [Gr96, 2.C] reveals the existence of a universal constant $C$ such that we have an asymptotically vanishing upper bound

$$
\frac{\text { sys }_{1}\left(\Sigma_{s}\right)^{2}}{\operatorname{area}\left(\Sigma_{s}\right)} \leq C \frac{(\log s)^{2}}{s}
$$

whenever $\Sigma_{s}$ is a closed, orientable surface of genus $s \geq 2$ with a Riemannian metric.

Inequality (5.8) admits the following higher-dimensional analogue [Ka02]. Let $n \in \mathbb{N}$ and consider the complex projective plane blown up at $n$ points, $\mathbb{C} P^{2} \# n \overline{\mathbb{C P}}^{2}$, where bar denotes reversal of orientation, while \# is connected sum. Assume that 
the Surjectivity conjecture for the period map is satisfied for such manifolds, namely every line in the positive cone of the intersection cone occurs as the selfdual direction of a suitable metric. Then the conformal 2-systole satisfies the bounds

$$
C^{-1} \sqrt{n}<\sup _{\mathbf{g}}\left\{\lambda_{1}\left(H^{2}\left(\mathbb{C} P^{2} \# n \overline{\mathbb{C P}}^{2}, \mathbb{Z}\right),||_{L^{2}}\right)\right\}^{2}<C n
$$

as $n \rightarrow \infty$, where $C>0$ is a numerical constant, the supremum is taken over all smooth metrics $\mathbf{g}$ on $\mathbb{C} P^{2} \# n \overline{\mathbb{C P}}^{2}$, and ||$_{L^{2}}$ is the norm associated with $\mathbf{g}$ by formula (5.6).

It would be interesting to eliminate the dependence of inequality (5.10) on the surjectivity conjecture. Moreover, can one improve the lower bound in (5.10) to linear dependence on $n$ ? Here one could try to apply an averaging argument, using Siegel's formula as in [MH73], over integral vectors satisfying $q_{n, 1}(v)=-p$. Here one seeks a vector $v \in \mathbb{R}^{n, 1}$ such that the integer lattice $\mathbb{Z}^{n, 1} \subset \mathbb{R}^{n, 1}$ has the Conway-Thompson behavior with respect to the positive definite form $S R\left(q_{n, 1}, v\right)$.

2. A conjectured Pu-times-Loewner inequality. We conjecture an optimal inequality for certain 4-manifolds $X$ with first Betti number $b_{1}(X)=2$ which can be thought of as a product of the inequalities of Loewner (2.3) and $\mathrm{Pu}(2.5)$. The main obstacle to its proof is the absence of a generalized Pu's inequality (2.7) (or conjecture 3.8 for $n=1$ ). For simplicity, we first state it for the manifold $\mathbb{T}^{2} \times \mathbb{R} P^{2}$, and later discuss the relevant topological hypothesis.

Conjecture 5.11. Assume (2.7). Then every metric $\mathbf{g}$ on $\mathbb{T}^{2} \times \mathbb{R} P^{2}$ satisfies the inequality

$$
\operatorname{sys}_{1}(\mathbf{g})^{2} \text { stsys }_{1}(\mathbf{g})^{2} \leq \frac{2}{\sqrt{3}} \frac{\pi}{2} \operatorname{vol}_{4}(\mathbf{g})
$$

while a metric which satisfies the boundary case of equality must admit a Riemannian submersion onto a Loewner-extremal torus, with fibers which are Pu-extremal, i.e. real projective planes of constant Gaussian curvature.

The relevant topological hypothesis is the following: $X$ should be a 4-manifold with $b_{1}(X)=2$, such that moreover the universal free abelian cover $\bar{X}$ is essential in dimension 2, cf. [KKS], so that in particular $X$ satisfies the nonvanishing condition $[\bar{X}] \neq 0 \in H_{2}\left(\bar{X}, \mathbb{Z}_{2}\right)$, where $[\bar{X}]$ is the Poincare dual of the pullback by $\overline{A J}_{X}$ of the fundamental class with compact support of the universal cover $\mathbb{R}^{2}$ of the Jacobi torus of $X$.

We conjecture that in the boundary case of equality, both the topology and the metrics must be right, namely: (a) the manifold $X$ must fiber smoothly over $\mathbb{T}^{2}$ with fiber $\mathbb{R} P^{2}$; (b) the Abel-Jacobi map $A J_{X}: X \rightarrow \mathbb{T}^{2}$ is a Riemannian submersion with fibers of constant volume.

3. An optimal inequality in dimension and codimension 1. Recently, V. Bangert and the second author [BanK03] clarified the constants involved in Gromov's inequalities mentioned at the beginning of section 5 , and showed in particular the following. Let $k>0$, and assume $H^{k}(X, \mathbb{R}) \neq 0$. Let $k=\sum k_{j}$ and 
assume that the group $H^{k}(X, \mathbb{R})$ is spanned by cup products of classes of dimensions $k_{j}$. Then

$$
\prod_{j} \text { stsys }_{k_{j}}(\mathbf{g}) \leq C(k)\left(\prod_{j} b_{k_{j}}(X)\left(1+\log b_{k_{j}}(X)\right)\right) \operatorname{stsys}_{k}(\mathbf{g}), \forall \mathbf{g}
$$

where the constant $C(k)$ depends only on $k$ (and not on the metric or the Betti numbers). On the other hand, the optimal constants in such inequalities are generally unknown, unlike Loewner's classical inequality (2.3).

The following sharp inequality generalizing Loewner's is proved in [Gr99, pp. 259-260], based on the techniques of [Bu-Iv95]. Assume that the dimension, first Betti number, and real cuplength of $X$ are all equal to $n$. Then

$$
\text { stsys }_{1}(\mathbf{g})^{n} \leq\left(\gamma_{n}\right)^{\frac{n}{2}} \operatorname{vol}_{n}(\mathbf{g}), \forall \mathbf{g}
$$

where $\gamma_{n}$ denotes the classical Hermite constant, while equality in (5.14) is attained precisely by flat tori whose deck transformations define the densest sphere packings in dimension $n$. An optimal stable systolic inequality, for $n$-manifolds $X$ with first Betti number $b_{1}(X, \mathbb{R})$ equal to one, is due to J. Hebda [He86]:

$$
\operatorname{stsys}_{1}(\mathbf{g}) \operatorname{sys}_{n-1}(\mathbf{g}) \leq \operatorname{vol}_{n}(\mathbf{g}),
$$

with equality if and only if $(X, \mathbf{g})$ admits a Riemannian submersion with connected minimal fibers onto a circle. An optimal inequality, involving the conformal 1systole, is proved in [BanK2], namely equation (5.17) below. The new inequality generalizes simultaneously Loewner's inequality (2.3), Hebda's inequality (5.15), the inequality [BanK03, Corollary 2.3], as well as certain results of G. Paternain [Pa01]. We define the Bergé-Martinet constant, $\gamma_{b}^{\prime}$, by setting

$$
\gamma_{b}^{\prime}=\sup _{L}\left\{\lambda_{1}(L) \lambda_{1}\left(L^{*}\right) \mid L \subset\left(\mathbb{R}^{b},||\right)\right\}
$$

where the supremum is over all lattices $L$ in $\mathbb{R}^{b}, c f$. [BeM89, CS94]. Here $L^{*}$ denotes the lattice dual to $L$, while || is a Euclidean norm. The supremum defining $\gamma_{b}^{\prime}$ is attained, and the lattices realizing it are called dual-critical [BeM89]. The following is proved in [BanK2]. Let $X$ be a compact, oriented, $n$-dimensional manifold with positive first Betti number $b_{1}(X) \geq 1$. Then every metric $\mathbf{g}$ on $X$ satisfies

$$
\operatorname{confsys}_{1}(\mathbf{g}) \operatorname{sys}_{n-1}(\mathbf{g}) \leq \gamma_{b_{1}(X)}^{\prime} \operatorname{vol}_{n}(\mathbf{g})^{\frac{n-1}{n}},
$$

where equality occurs if and only if there exists a dual-critical lattice $L$ in Euclidean space $\mathbb{R}^{b}$ ( $c f$. formula (5.16)), and a Riemannian submersion of $X$ onto the flat torus $\mathbb{R}^{b} / L$, such that all fibers are connected minimal submanifolds of $X$.

\section{Isoembolic Inequalities}

Let $\operatorname{inj}(X)$ represent the injectivity radius of a Riemannian manifold $X$. All geodesics of length $\leq i n j(X)$ will thus be minimizing. This section has to do with "isoembolic inequalities" which are estimates of volume in terms of the injectivity radius. In the closed ball, $B_{p}(r)$, centered at $p$ of radius $r<\frac{\operatorname{inj}(X)}{2}$ all geodesic segments will minimize and it will be $S G M$ as a manifold with boundary (which we denote $S(r)$ ). We will let $\omega_{n}$ represent the volume of the standard unit sphere (of injectivity radius and diameter $\pi$ ) while $\beta_{n}$ will be the volume of the Euclidean $n$-ball (so that $n \beta_{n}=\omega_{n-1}$ ). One major open question is: 


\section{Conjecture 6.1.}

a) For any $r \leq \frac{i n j(X)}{2}, \operatorname{vol}(B(r)) \geq \frac{\omega_{n}}{2}\left(\frac{2}{\pi}\right)^{n} r^{n}$ with equality holding only if the ball is isometric to a hemisphere.

b) For any $r \leq i n j(X), \operatorname{vol}(B(r)) \geq \frac{\omega_{n}}{\pi^{n}} r^{n}$, where equality holds if and only if $X$ is isometric to the round sphere of injectivity radius $r$ (i.e. extrinsic radius $\frac{r}{\pi}$ ).

In part a) above, the hemisphere in the equality case will be that of a sphere of (extrinsic) radius $\frac{2 r}{\pi}$ (so the hemisphere is a metric ball of intrinsic radius $r$ ). In fact a) is a stronger conjecture than b) since by the triangle inequality $B(r)$ contains two balls of radius $\frac{r}{2}$ with disjoint interiors.

1. Berger's 2 and 3 dimensional estimates. The early results on this conjecture and the conjecture itself are due to Berger (see [Be77]). The known results take the form of proving inequalities $\operatorname{vol}(B(r)) \geq c(n) r^{n}$ for non-sharp constants $c(n)$. In 2 dimensions it is not hard to get such an estimate when $r \leq$ $\frac{i n j(X)}{2}$. Simply notice that for all $t \leq r, \operatorname{vol}\left(S_{p}(t)\right) \geq 4 t$ (i.e. the length of the boundary of $B_{p}(t)$ is $\geq 4 t$ ) since "antipodal" points on $S(t)$ must be at least $2 t$ apart in $X$ and hence also along $S(t)$. This says that $\operatorname{vol}\left(B_{p}(r)\right)=\int_{0}^{r} \operatorname{vol}\left(S_{p}(t)\right) d t \geq 2 r^{2}$. The conjecture would say $\operatorname{vol}(B(r)) \geq \frac{8}{\pi} r^{2}$ and hence this simple argument leads to a constant not too far from the best possible. The conjecture with the sharp constant is still open. (Using ideas in [C-D] - cf. section 2 - one can show in this 2-dimensional setting that $\operatorname{vol}\left(B_{p}(t)\right) \geq 4 \pi-\operatorname{vol}\left(S_{p}(t)\right)$ and then use this to get the better estimate $\operatorname{vol}\left(B_{p}(r)\right) \geq \frac{16}{4+\pi} r^{2}$, but since this argument is a little involved and does not yield the sharp constant we will not pursue it.)

An interesting example to keep in mind in the "Mercedes-Benz" example. Consider three unit length line segments in the plane emanating from the origin making angles of $\frac{2 \pi}{3}$. Thin tubular neighborhoods have arbitrarily small area but every boundary point is at a bounded distance from its antipodal point (the point halfway around the boundary). Thus one needs more than just bounded distance between "antipodal boundary points" to get area lower bounds.

One might hope to generalize the above simple argument for the area of 2-balls by showing:

Question 6.2. Let $\mathbf{g}$ be a Riemannian metric on the $n$-sphere $S^{n}$ such that the g-distance between antipodal points is $\geq t$.

a) Is $\operatorname{vol}\left(\left(S^{n}, \mathbf{g}\right)\right) \geq \frac{\omega_{n}}{\pi^{n}} t^{n}$ ?

b) Is there any constant $c(n)$ such that $\operatorname{vol}\left(\left(S^{n}, \mathbf{g}\right)\right) \geq c(n) t^{n}$ ?

In the above one can either think of the standard antipodal map when $S^{n}$ is embedded in $\mathbb{R}^{n}$ in the standard way, or simply take the antipodal map to be any order two fixed point free diffeomorphism. Metric balls of radius $\frac{t}{2}$ satisfy this when $t \leq \operatorname{inj}(X)$.

The easy argument we gave above for the volume of 2-balls consists of answering a) above in the case $n=1$. This is the only case where a) is known. Berger in [Be77] gave an answer to b) above for $n=2$ and hence showed by integrating (as in the 2 dimensional argument) that there is a constant $c$ such that for 3 dimensional manifolds when $r \leq \frac{\operatorname{inj}(X)}{2}$, then $\operatorname{vol}\left(B_{p}(r)\right) \geq c r^{3}$. 
Though part a) of Question 6.2 in 2 dimensions is still open (and very interesting), there are enough reasons to believe it to be true that it would warrant being called a conjecture. One approach would be to use the uniformization theorem and conformal length techniques (but note that the antipodal map need not be conformal).

In higher dimensions there is not enough evidence one way or the other even for question b). However (see [Cr02]) there are constants such that for any given metric $g$ on the 3-sphere either Question $6.2 \mathrm{~b}$ ) holds or Question 2.11 holds. This follows from a lower bound on the filling radius ( $c f$. section 1) in terms of the infimum of the length of closed curves who link their antipodal images along with an application of Theorem 3.1. In the other direction, Ivanov (see [Iv97] or [Iv98]) has given examples of a sequence of metrics on $S^{3}$ that Gromov-Housdorff converge to the standard metric but whose volumes go to zero. Although this does not give a counter example it does show that the topological properties of the antipodal map are important in the above question. (Even in two dimensions long thin cigar shapes show that simply because every point is far from some other point the area need not be large.)

2. Higher dimensions. The two ways that higher dimensional isoembolic type inequalities have been proved are via Gromov's estimate of section 1 and via an estimate of Berger and Kazdan [Be-Ka78]. The Berger-Kazdan inequality first appeared in the proof of the Baschke conjecture for spheres (see [Bes78] appendices $\mathrm{D}$ and $\mathrm{E})$. Berger then used the result to give the sharp estimate which holds for any compact Riemannian manifold:

$$
\operatorname{vol}(X) \geq \frac{\omega_{n}}{\pi^{n}} \operatorname{inj}(X)^{n}
$$

with equality holding only for round spheres. If you know that $X$ is not homeomorphic to a sphere then you can do better $[\mathbf{C r 8 8 A}]$, showing $\operatorname{vol}(X) \geq c(n) \operatorname{inj}(X)^{n}$ where $c(n)$ is an explicit constant larger than $\frac{\omega_{n}}{\pi^{n}}$.

The Berger-Kazdan inequality along with Santaló's formula [San52, San76, Chapter 19] was used in [Cr80] to give a general sharp isoperimetric inequality for compact Riemannian manifolds, $X$, with boundary, $\partial X$ where all geodesic minimize (e.g. $S G M$ manifolds):

$$
\frac{\operatorname{vol}(\partial X)}{\operatorname{vol}(X)^{\frac{n-1}{n}}} \geq C(n)
$$

where the constant $C(n)$ is just $\omega_{n-1}\left(\frac{2}{\omega_{n}}\right)^{\frac{n-1}{n}}$. Equality holds if and only if $X$ is isometric to a hemisphere. (The inequality has a version for all manifolds with boundary which involves a term measuring the fraction of geodesics that minimize to the boundary.) One gets a (non-sharp) version of conjecture 6.1 a) in all dimensions by applying this to the balls $B_{p}(t)$ and integrating $t$ from 0 to $r$ :

$$
\operatorname{vol}\left(B_{p}(r)\right) \geq C(n)^{n} r^{n} .
$$

Further, applying the isoperimetric inequality again we see: $\operatorname{vol}\left(S_{p}(r)\right) \geq C(n)^{n} r^{n-1}$ The failure of this estimate to be sharp comes from the fact that the isoperimetric inequality is not sharp for spherical caps (i.e. metric balls in the round sphere) unless they are hemispheres. We mentioned before (Conjecture 6.1 part a) that, for $r \leq \frac{i n j}{2}$, we do not know the optimal lower bound for $\operatorname{vol}\left(B_{p}(r)\right)$. Except in case of dimension 2, we also don't know the optimal lower bound for $\operatorname{vol}\left(S_{p}(r)\right)$, however 
the above isoperimetric inequality will imply the sharp estimate on $\operatorname{vol}\left(S_{p}(r)\right)$ if Conjecture 6.1 part a is proven.

Although we are unable to prove Conjecture 6.1 for all balls there are some results about the "average" volume of balls, AveVol(r), of a compact Riemannian manifold $X$. By this we mean

$$
\operatorname{AveVol}(r) \equiv \frac{1}{\operatorname{vol}(X)} \int_{X} \operatorname{vol}\left(B_{p}(r)\right) d p
$$

where $d p$ is the Riemannian volume form. In [Cr84] it is shown for any $r \leq$ $\operatorname{inj}(X)$ that $\operatorname{AveVol}(r) \geq \frac{\omega_{n}}{\pi^{n}} r^{n}$ with equality holding only for the round sphere of injectivity radius $r$. Thus showing that part b) of Conjecture 6.1 is true "on the average" (and giving another proof of Berger's isoembolic inequality 6.3). If one further knows that $X$ has no conjugate points, then one can improve this to $\operatorname{AveVol}(r) \geq \beta_{n} r^{n}$, where equality holds if and only if $X$ is flat [Cr92]. In fact, this holds for all $r$, if one interprets $\operatorname{vol}\left(B_{p}(r)\right)$ to mean the volume of a ball in the universal cover centered at a lift of $p$.

Gromov in [Gr83], showed (in an argument similar to the one presented in section 1) that the filling radius of a compact Riemannian manifold is always bounded from below by a constant times the injectivity radius. This, along with Theorem 3.1 , gave an alternative proof to Berger's isoembolic inequality 6.3 (albeit with a non-sharp constant). This idea was extended in [G-P92] to get non-sharp universal estimates on the volumes of balls in terms of the local geometric contractibility function. As a special case, it gives an alternative proof of inequality 6.4. Sabourau also used these filling radius ideas in [Sab2] to give improved versions of both (6.3) and (6.4) (with worse constants) where instead of injectivity radius he is able to substitute the length of the shortest geodesic loop. A geodesic loop is a closed curve which is a geodesic at all but one point. Geodesic loops are much more abundant than closed geodesics and the shortest one will have length, $\operatorname{sgl}(\mathbf{g})$, which satisfies

$$
2 \operatorname{inj}(\mathbf{g}) \leq \operatorname{sgl}(\mathbf{g}) \leq L(\mathbf{g})
$$

Sabourau's version of (6.4), which holds for all $r \leq \frac{1}{2} \operatorname{sgl}(\mathbf{g})$, also used the ideas of [G-P92].

\section{Acknowledgments}

We are grateful to E. Calabi, I. Chavel, A. Nabutovsky, and S. Sabourau for reading a draft version of the manuscript and for their comments.

\section{References}

[Ac60] R. D. M. Accola, Differential and extremal lengths on Riemannian surfaces, Proc. Math. Acad. Sci. USA 46 (1960), 83-96.

[Ak02] H. Akrout, Théorème de Voronoï dans les espaces symétriques. [Voronoi theorem in symmetric spaces] Canad. J. Math. 54 (2002), no. 3, 449-467.

[A162] F. Almgren Jr., The homotopy groups of the integral cycle groups, Topology 1 (1962),257-299.

[Ar79] M. A. Armstrong, Basic topology. McGraw-Hill Book Co. (UK), Ltd., London-New York, 1979.

[Bab02] I. Babenko, Forte souplesse intersystolique de variétés fermées et de polyèdres, Annales de l'Institut Fourier 524 (2002), 1259-1284.

[BabK98] I. Babenko and M. Katz, Systolic freedom of orientable manifolds, Annales Scientifiques de l'E.N.S. (Paris) 31 (1998), 787-809. 
[BKS98] I. Babenko, M. Katz, and A. Suciu, Volumes, middle-dimensional systoles, and Whitehead products, Math. Res. Lett. 5 (1998), no. 4, 461-471.

[Ban94] V. Bangert, Geodesic rays, Busemann functions and monotone twist maps, Calc. Var. Part. Diff. Eq. 2 (1994), no. 1, 49-63.

[BanK03] V. Bangert and M. Katz, Stable systolic inequalities and cohomology products, Comm. Pure Appl. Math. 56 (2003), in press. math.DG/0204181

[BanK2] V. Bangert, and M. Katz, Riemannian manifolds with harmonic 1-forms of constant norm, in preparation.

[Bav86] C. Bavard, Inégalité isosystolique pour la bouteille de Klein, (French) [Isosystolic inequality for the Klein bottle] Math. Ann. 274 (1986), no. 3, 439-441.

[Bav92a] C. Bavard, Ingalits isosystoliques conformes, Comment. Math. Helv. 67 (1992), no. 1, 146-166.

[Bav92b] C. Bavard, La systole des surfaces hyperelliptiques, Prepubl. Ec. Norm. Sup. Lyon 71 (1992).

[BeM89] A.-M. Bergé, and J. Martinet, Sur un problème de dualité lié aux sphères en géométrie des nombres, J. Number Theory 32 (1989), 14-42.

[Be77] M. Berger, Volume et rayon d'injectivité dans les variétés riemanniennes de dimension 3, Osaka J. Math., 14 (1977), 191- 200.

[Be80] M. Berger, Une bourne inférieure pour le volume d'une variété riemannienne en function du rayon d'injectivité, Ann. Inst. Fourier (Grenoble) 30 (1980) no.3, 259-265.

[Be83] M. Berger, Filling Riemannian manifolds or isosystolic inequalities, Global Riemannian Geometry, Ellis Horwood Ser. Math. Appl. (1983), 75-84.

[Be-Ka78] M. Berger and J. L. Kazdan, A Sturm-Liouville inequality with applications to an isoperimetric inequality for volume in terms of injectivity radius, and to Wiedersehen manifolds, in General Inequalities 2 (Proceedings of the second International Conference on General Inequalities, 1978), E. F. Beckenbach (ed.), ISNMA47, Birkhauser Verlag, no.3, 251-254.

[Bes78] A. Besse. Manifolds all of whose geodesics are closed, Ergebisse Grenzgeb. Math., no. 93, Springer, Berlin, 1978.

[B-C-G95] G. Besson, G. Courtois, and S. Gallot, Entropies et rigidités des espaces localement symétriques de courbure strictement négative, Geom. Funct. Anal. 5 (1995) no. 5, 731-799.

[B-C-G96] G. Besson, G. Courtois, and S. Gallot, Minimal entropy and Mostow's rigidity theorems, Ergodic Theory Dynam. Systems 16 (1996) no. 4, 623-649.

[Bi27] G. D. Birkhoff, Dynamical systems, Amer. Math. Soc. Colloq. Publ. Vol. 9, Providence, RI, 1927.

[B161] C. Blatter, Über extremallängen auf geschlossen flächen, Comment. Math. Helv. 35 (1961), no.3, 153-168.

[Bo91] F. Bonahon, The geometry of Teichmüller space via geodesic currents, Inven. Math. 33 (1991), 445-464.

[Bo93] F. Bonahon, Surfaces with the same marked length spectrum, Topology Appl. 50 (1993) no. 1, 55-62.

[Br96] R. Bryant, On extremals with prescribed Lagrangian densities Manifolds and geometry (Pisa, 1993), 86-111, Sympos. Math., XXXVI, Cambridge Univ. Press, Cambridge, 1996.

[Bu-Iv95] Burago, D.; Ivanov, S.: On asymptotic volume of tori, Geom. Funct. Anal. 5 (1995), no. $5,800-808$.

[Bu-Iv2] D. Burago and S. Ivanov, On asymptotic volume of Finsler tori, minimal surfaces in normed spaces, and symplectic filling volume, (preprint).

[B-Z80] Yu. Burago and V. Zalgaller, Geometric Inequalities, "Nauka"(1980), Russian.

[B-Z88] Yu. Burago and V. Zalgaller, Geometric Inequalities, Springer (1988).

[BuSa94] P. Buser, and P. Sarnak, On the period matrix of a Riemann surface of large genus. With an appendix by J. H. Conway and N. J. A. Sloane, Invent. Math. 117 (1994) no. 1, 27-56.

[Ca96] E. Calabi, Extremal isosystolic metrics for compact surfaces, Actes de la table ronde de geometrie differentielle, Sem. Congr. 1 (1996), Soc. Math. France 165-204.

[Ca03] E. Calabi, private conversations.

[Ca3] E. Calabi, On the Extremal Isosystolic Problems, in preparation. 
[CaCa92] E. Calabi and J. Cao, simple closed geodesics on convex surfaces, J. Diff. Geom., 36 (1992), 517-549

[Ch93] I. Chavel, Riemannian geometry - a modern introduction. Cambridge Tracts in Mathematics, 108. Cambridge University Press, Cambridge, 1993.

[CS94] J. H. Conway; N. J. A. Sloane: On lattices equivalent to their duals. J. Number Theory 48 (1994), no. 3, 373-382.

[Cr80] C. Croke, Some isoperimetric inequalities and eigenvalue estimates, Ann. Scient. Ec. Norm. Sup., 4e serie, t.13 (1980), 419-435.

[Cr84] C. Croke, Curvature Free Volume Estimates, Inventiones Mathematicae 76 (1984), 515-521.

[Cr88A] C. Croke, An Isoembolic Pinching Theorem, Invent. Math., 92 (1988), 385-387.

[Cr88B] C. Croke, Area and the Length of the Shortest Closed Geodesic, J. Diff. Geom. 27 (1988), 1-21.

[Cr90] C. Croke, Rigidity for surfaces of non-positive curvature, Comm. Math. Helv. 65 (1990) no.1, 150-169.

[Cr91] C. Croke, Rigidity and the distance between boundary points, J. Diff. Geom. 33 (1991), 445-464.

[Cr92] C. Croke, Volumes of balls in manifolds without conjugate points, International J. Math. 3 (1992) no.4, 455-467.

[Cr01] C. Croke, Rigidity Theorems in Riemannian geometry, (preprint - to appear in "Geometry, Inverse Problems and Control of PDE" based on the conference at IMA, July 2001).

[Cr02] C. Croke, the volume and lengths on a three sphere, Comm. Anal. Geom., 10 (2002) no. $3,467-474$.

[C-D] C. Croke, N. Dairbekov, Lengths and volumes in Riemannian manifolds, preprint.

[C-D-S00] C. Croke, N. Dairbekov, and V. Sharafutdinov, Local boundary rigidity of a compact Riemannian manifold with curvature bounded above, Trans. Amer. Math. Soc. 325 (2000) no.9, 3937-3956.

[C-F-F92] C. Croke, A. Fathi, and J. Feldman, The marked length-spectrum of a surface of nonpositive curvature, Topology 31 (1992) no. 4, 847-855.

[FK92] H. M. Farkas, I. Kra, Riemann surfaces. Second edition. Graduate Texts in Mathematics, 71. Springer-Verlag, New York, 1992.

[Fe74] H. Federer, Real flat chains, cochains, and variational problems. Indiana Univ. Math. J. 24 (1974), 351-407.

[Fr99] M. Freedman, $\mathbb{Z}_{2}$-systolic-freedom, Proceedings of the Kirbyfest (Berkeley, CA, 1998), 113-123, Geom. Topol. Monogr., vol. 2, Geom. Topol, Coventry, 1999.

http://www.maths . warwick.ac.uk/gt/GTMon2/paper6.abs.html.

[Gr81] M. Gromov, Structures métriques pour les variétés riemanniennes. (French) [Metric structures for Riemann manifolds] Edited by J. Lafontaine and P. Pansu. Textes Mathématiques [Mathematical Texts], 1. CEDIC, Paris, 1981.

[Gr83] M. Gromov, Filling Riemannian manifolds, J. Diff. Geom. 18 (1983), 1-147.

[Gr96] M. Gromov, Systoles and intersystolic inequalities Actes de la Table Ronde de Géométrie Différentielle (Luminy, 1992), 291-362, Sémin. Congr., vol. 1, Soc. Math. France, Paris, 1996.

www .emis.de/journals/SC/1996/1/ps/smf_sem-cong-1_291-362.ps.gz

[Gr99] M. Gromov, Metric structures for Riemannian and non-Riemannian spaces, Progr. in Mathematics, vol 152, Birkhäuser, Boston, 1999.

[G-P92] R. Green and P. Petersen, Little topology, big volume, Duke Math. J. 67 (1992), no. 2, 273-290.

[Ha92] U. Hamenstädt, Time-preserving conjugacies of geodesic flows, Ergod. Th. \& Dynam. Sys. 12 (1992), 67-74.

[Ha99] U. Hamenstädt, Cocycles, symplectic structures and intersection, Geom. Funct. Anal. 9 (1999) no. 1, 90-140.

[He82] J. Hebda, Some lower bounds for the area of surfaces, Invent. Math. 65 (1982), 485491.

[He86] J. Hebda, The collars of a Riemannian manifold and stable isosystolic inequalities, Pacific J. Math. 121 (1986), 339-356. 
[Iv02] S. Ivanov, On two-dimensional minimal fillings, St. Petersbg. Math. J. 13 (2002), no. $1,17-25$.

[Iv97] S. Ivanov Gromov-Hausdorff Convergence and volumes of manifolds, Algebra i Analiz, 9 (1997) No.5, 65-83 (Russian).

[Iv98] S. Ivanov, Gromov-Hausdorff Convergence and volumes of manifolds, St. Petersburg Math. J., 9 (1998) No.5, 945-959.

[Ka83] M. Katz, The filling radius of two-point homogeneous spaces, J. Diff. Geom. 18 (1983) 505-511.

[Ka91A] M. Katz, On neighborhoods of the Kuratowski imbedding beyond the first extremum of the diameter functional, Fundamenta Mathematicae 137 (1991) 15-29.

[Ka91B] M. Katz, The rational filling radius of complex projective space, Topology and Its Applications 42 (1991) 201-215.

[Ka91C] M. Katz, Pyramids in the complex projective plane, Geometriae Dedicata 40 (1991) 171-190.

[Ka95] M. Katz, Counterexamples to isosystolic inequalities, Geom. Dedicata 57 (1995), 195206.

[Ka02] M. Katz, Local calibration of mass and systolic geometry, Geometric and Functional Analysis 12, issue 3 (2002), 598-621.

[Ka3] M. Katz, Four-manifold systoles and surjectivity of period map, Comment. Math. Helvetici (2003), conditionally accepted for publication.

[KS99] M. Katz, and A. Suciu, Volume of Riemannian manifolds, geometric inequalities, and homotopy theory, in Tel Aviv Topology Conference: Rothenberg Festschrift (M. Farber, W. Lück, and S. Weinberger, eds.), Contemp. Math., vol. 231, AMS (1999), pp. 113136.

[KS01] M. Katz, and A. Suciu, Systolic freedom of loop space, Geometric and Functional Analysis 11 (2001), 60-73.

[KKS] M. Katz, M, Kreck, \& A. Suciu, Free abelian covers, short loops, stable length, and systolic inequalities. arXiv:math.DG/0207143

[Ko87] S. Kodani, On two-dimensional isosystolic inequalities Kodai Math. J. 10 (1987) no. 3, 314-327.

[Mae94] M. Maeda, The length of a closed geodesic on a compact surface, Kyushu J. Math., 48:1 (1994), 9-18.

[Mas97] D. Massart, Stable norms of surfaces: local structure of the unit ball of rational directions. Geom. Funct. Anal. 7 (1997), no. 6, 996-1010.

[MH73] J. Milnor and D. Husemoller, Symmetric bilinear forms. Springer, 1973.

[N-R02] A. Nabutovsky and R. Rotman, The length of the shortest closed geodesic on a 2dimensional sphere, Int. Math. Res. Not. (2002) no. 23, 1211-1222.

[N-R1] A. Nabutovsky and R. Rotman, Upper bounds on the length of a shortest closed geodesic and quantitative Hurewicz theorem, (preprint).

[N-R2] A. Nabutovsky and R. Rotman, Volume, diameter, and the minimal mass of a stationary 1-cycle, (preprint).

[Ot90A] J.-P. Otal, Le spectre marqué des longueurs des surfaces à courbure négative, Ann. of Math. 131 (1990), 151-162.

[Ot90B] J.-P. Otal, Sur les longueurs des géodésiques d'une métrique à courbure négative dans le disque, Comment. Math. Helv, 65 (1990) no. 2, 334-347.

[Pa01] G. P. Paternain, Schrödinger operators with magnetic fields and minimal action functionals, Israel J. Math. 123 (2001), 1-27.

[Pitte97] C. Pittet, Systoles on $S^{1} \times S^{n}$, Differential Geom. Appl. 7 (1997), 139-142.

[Pitts81] J. Pitts, Existence and regularity of minimal surfaces on Riemannian manifolds, Ann. Math. studies 27(1981), Princeton University Press.

[Pu52] P.M. Pu, Some inequalities in certain nonorientable Riemannian manifolds, Pacific J. Math. 2 (1952), 55-71.

[Sab1] S. Sabourau, Filling radius and short closed geodesics of the sphere, preprint (2002).

[Sab2] S. Sabourau, Global and local volume bounds and the shortest geodesic loop, preprint (2002).

[Sab02c] S. Sabourau, Systoles des surfaces plates singulières de genre deux, preprint (2002).

[Sak88] T. Sakai, A proof of the isosystolic inequality for the Klein bottle, Proc. Amer. Math. Soc. 104 (1988) no. 2, 589-590. 
[San52] L. A. Santaló, Measure of sets of geodesics in a Riemannian space and applications to integral formulas in elliptic and hyperbolic spaces, Summa Brasil. Math. 3, (1952). 1-11.

[San76] L. A. Santaló, Integral Geometry and Geometric Probability. With a Foreword by Mark Kac. Encyclopedia of Mathematics and its Applications, Vol. 1. Addison-Wesley Publishing Co., Reading, Mass.-London-Amsterdam, 1976.

[Sch93] P. Schmutz, Riemann surfaces with shortest geodesic of maximal length. Geom. Funct. Anal. 3 (1993), no. 6, 564-631.

[S-Y94] R Schoen and S.-T. Yau, Lectures on Differential Geometry, International Press (1994).

[Si90] K. Sigmund, On the space of invariant measures for hyperbolic flows, Ann. of Math. 131(1990), 151-162.

[Ta92] P. M. Tamrazov, Methods for studying extremal metrics and moduli in a twisted Riemannian manifold (Russian. Russian summary) Mat. Sb. 183 (1992), no. 3, 55-75; translation in Russian Acad. Sci. Sb. Math. 75 (1993), no. 2, 333-351.

[Tre85] A. Treibergs, Estimates of volume by the length of shortest closed geodesics on a convex hypersurface, Invent. Math., 80 (1985), 481-488.

[Tro86] M. Troyanov, Les surfaces euclidiennes á singularités coniques. (French) [Euclidean surfaces with conical singularities] Enseign. Math. (2) 32 (1986), no. 1-2, 79-94.

[Wi92] F. Wilhelm, On the filling radius of positively curved manifolds. Invent. Math. 107 (1992), no. 3, 653-668.

[Ya82] S.-T. Yau, Problem section in "Seminar in Differential Geometry", ed. by S.-T. Yau, Princeton University Press (1982).

Christopher B Croke:

Department of Mathematics

University of Pennsylvania

Philadelphia, PA 19104-6395

ccroke@math.upenn.edu

Mikhail G. Katz

Department of Mathematics and Statistics

Bar Ilan University

Ramat Gan 52900, Israel

katzmik@math.biu.ac.il 
